Research Article

\title{
Multiphysics Modeling of Gas Turbine Based on CADSS Technology
}

\author{
Tian Wang $(\mathbb{D}$, Ping Xi, and Bifu Hu \\ School of Mechanical Engineering and Automation, Beihang University, Beijing 100192, China \\ Correspondence should be addressed to Tian Wang; wangt703@buaa.edu.cn
}

Received 12 September 2020; Revised 29 September 2020; Accepted 30 September 2020; Published 23 October 2020

Academic Editor: Chaoqun Duan

Copyright (C) 2020 Tian Wang et al. This is an open access article distributed under the Creative Commons Attribution License, which permits unrestricted use, distribution, and reproduction in any medium, provided the original work is properly cited.

Product modeling has been applied in product engineering with success for geometric representation. With the application of multidisciplinary analysis, application-driven models need specific knowledge and time-consuming adjustment work based on the geometric model. This paper proposes a novel modeling technology named computer-aided design-supporting-simulation (CADSS) to generate multiphysics domain models to support multidisciplinary design optimization processes. Multiphysics model representation was analyzed to verify gaps among different domain models' parameters. Therefore, multiphysics domain model architecture was integrated by optimization model, design model, and simulation model in consideration of domain model's parameters. Besides, CADSS uses requirement space, domain knowledge, and software technology to describe the multidisciplinary model's parameters and its transition. Depending on the domain requirements, the CADSS system extracts the required knowledge by decomposing product functions and then embeds the domain knowledge into functional features using software technology. This research aims to effectively complete the design cycle and improve the design quality by providing a consistent and concurrent modeling environment to generate an adaptable model for multiphysics simulation. This system is demonstrated by modeling turbine blade design with multiphysics simulations including computational fluid dynamics (CFD), conjugate heat transfer (CHT), and finite element analysis (FEA). Moreover, the blade multiphysics simulation model is validated by the optimization design of the film hole. The results show that the high-fidelity multiphysics simulation model generated through CADSS can be adapted to subsequent simulations.

\section{Introduction}

Computer-aided design (CAD) and computer-aided engineering (CAE) have been ubiquitously used in product development. However, design and analysis have been performed as two separate modules. According to product performance requirements, mechanical products' structure was designed through modeling software. The simulation analysis verifies whether the designed structure meets the requirements in dedicated simulation analysis software. Effectively integrating design and analysis processes can shorten product development cycle time and reduce the times of expensive physical experiments [1].

In general, different tools were utilized to build different models for product development in different domains during product development. The differences in both tools and expertise forced the analyst to preprocessing the CAD model, and any modifications made to the CAD model would cause an additional tedious geometry preparation [2]. Evidently, whether it is for process development or feature recognition in manufacturing automation, CAD modeling pays more attention to lower-level geometry and geometric parameters [3] and does not retain performance parameters and analysis parameters. The digital design does not pay much attention to the subsequent simulation, which is especially crucial for the preprocessing of the simulation analysis model and the modification of the simulation results [4]. The model is verified by the qualitative or quantitative performance parameter calculation of the simulation analysis. The lower-level geometric information operation is not intuitive for the analyst, and the qualitative or quantitative analysis results need the analyst to perform the 
corresponding conversion [5]. Subsequently, the designer can modify the CAD model, especially when it comes to the multidisciplinary fields such as dynamics, aerodynamics, structure, and thermodynamics. The modification requirements of the model are more frequent and complicated [6].

Therefore, multidisciplinary design optimization (MDO) approach is used to solve problems concerning multiple disciplines or subsystems [7]. Multiple disciplines or subsystems typically use analytical tools in their respective areas of expertise and establish a multiphysics simulation model that simulates the performance and behavior of the overall integrated design [8]. However, sophisticated engineering modeling across multiple domains is a daunting task. First, it takes a lot of time and effort to build a single discipline or domain simulation model. When it comes to multiphysics simulation model, multiple integrated information from many disciplines needs to be dealt with [9]. Second, highfidelity geometry information and other detailed configuration parameters are required when using computational fluid dynamics (CFD) or finite element method (FEM), even conjugated heat transfer (CHT) [10]. Then, the delay in creating a single subject analysis model by manual adjustment may slow down the entire MDO process [11]. Moreover, multidisciplinary optimization typically sets one or more goals [12]. When the design goals change, all simulation models should be updated synchronously rather than manually.

Product design essentially needs to meet the structural and performance requirements at the same time [12], but the existing feature modeling technology lacks support for product structure performance analysis, and performance information cannot be reused in the feature-based modeling method, especially for multiphysics domain modeling [13]. The problem leads to repeated and cumbersome CAE analysis, which significantly affects the design efficiency. In the existing product design and analysis process, the preprocessing is time-consuming, such as the conversion of the CAD model to CAE model through simplification and dimensionality reduction method [14], defining material properties and loading boundary conditions (displacement constraints and loads) [15]. Moreover, most of the above processes directly operate geometric models based on B-rep expression.

In order to improve the efficiency of preprocessing, some scholars introduced feature technology in the field of CAE analysis and proposed the concept of analysis features. Nizar et al. [16] present a representation method for mechanical analysis features, but this method is only suitable for engineering analysis in the early conceptual design stage of the product, and it is challenging to express structural performance analysis features. Shephard et al. [17] used different parts of the CAD model as the analysis features and used them as the basic unit of the model simplification process. This method brings convenience to the simplification of the model, but it is challenging to support other processing related to the analysis. Lee et al. [18] simplified the design features [19] as an analysis feature and proposed an integrated model for the unified expression of design and analysis features. This model can derive different levels of geometry and analysis models according to different needs. This method requires the analysis features to be mapped one-to-one with the design features. However, such mappings do not necessarily exist, making it difficult to handle complex features. Aifao et al. [20] used a set of analysis processes as analysis features and proposed a physical model including information such as load distribution, a simulation model that can evaluate physical model methods, and an observation model that can quantitatively evaluate product behavior. This method can achieve the reuse of analytical features to a certain extent. However, the lack of fixed template in engineering analysis leads to low flexibility [21].

For the CAD-CAE integration solution, the basic idea is to integrate the modeling and analysis module using unified software [22]. Through the object-linking technology (OTL) of commercial software, the CAD system can directly call the built-in CAE calculation module, such as CATIA module and NX and MSC solvers. On the one side, the modeling and analysis integration interface is unified and no intermediate model conversion is required. On the other side, the analysis module integrated under the CAD system platform has limited solution ability and cannot handle complex analysis problems.

Standard exchange files can be utilized to carry out the model between platforms [23], such as DXF, STEP, and IGES. The common data model (CDM) modeling and analysis integration uses the method of customizing intermediate files and deals with the data exchange problem of modeling and simulation platform [15]. A unified integration platform can guarantee the data compatibility between the CAD model and CAE model to ensure the flexibility of selecting the modeling and simulation platform [24]. However, the standard file interface sacrificed high-level design information such as features to ensure simplicity and versatility. Resulted in loss of model data, standard files cannot meet the relationship between the CAD model and CAE model. Consequently, it is challenging to implement the analysis and verification process of frequent modification iteration automatically or semiautomatically. Constructing the intermediate platform method analyzes the CAD model and the CAE model and realizes the data exchange through the intermediate model. To a certain extent, the CAD model can be transmitted to change the associated process of the CAE model, and the proprietary algorithm and program design are realized. However, model management and data exchange rely heavily on expert experience and the development of special algorithms. These limitations result in the lack of universality in the process of designanalysis integration.

In terms of integrated framework solutions, Peak et al. [25] proposed a multirepresentation architecture (MRA) to address data sharing between CAD and CAE applications. Sudarsan et al. [26] proposed a design-analytical integration framework based on the concept of PLM, using the expression of the main model and functional model to realize the construction of the framework model. Smit et al. [4] realized the association between design and analysis models by extending the multiview feature-based modeling concept 
to the paradigm of the analysis domain. Kim et al. [14] proposed a simplified method based on the feature-based boundary representation (B-rep) model, which uses a sequential iterative volume decomposition. The method generates a feature-based model from the B-rep model by applying volume decomposition methods sequentially and iteratively. Gujarathi et al. [15] proposed the integration of design-analysis using a unified data model. Li et al. [27] present a practical CAD/CFD integration framework that seamlessly integrates CAD and CFD tools to facilitate the cycle product development process and simplify the CFD solver setup.

In addition, Iso-geometric Analysis (IGA) in view of tighter CAD-CAE integration considered the whole geometric model directly as the analysis model using the same basis function to express the CAD geometry [28], and the geometric model can be directly used for analysis and design based on common data [29]. Simulation calculations and model modifications can be completed in one step, instead of having to undergo complex model processing and grid discretization like the current popular finite element method [30], thereby effectively reducing the design grid discretization [31]. However, these design attempts are only experimental and have not been applied to industrial practice at present.

The above literature concerns the construction of designanalysis integration framework and the analysis feature modeling method. Few researchers pay attention to the combination of the preprocessing stage and CAD modeling for the CAE process. The application of manufacturingoriented product models to numerical simulation relies mainly on manual processing, while the integration of CAD modeling and CAE analysis focuses on constructing an integrated framework for modeling and analysis. Dealing with modeling product in multiphysics simulation modeling is not discussed and researched.

Due to the complex dependencies and lack of management tools [32], it is challenging to guarantee model integrity and adaptability. Ideally, analysts only spend time on analysis work rather than these repetitive preprocessing tasks. A rapid parameter configuration method for two dimensions simplified model was generated for pipe-net calculation [33], and the results obtained are in good agreement with the results of the three-dimensional model simulation [34]. Therefore, in order to effectively complete the design cycle and improve the design quality, it is necessary to establish a reasonable modeling technology to ensure that the CAD model of the design can adapt to the subsequent simulation, and the analysis results of the numerical simulation can be quickly fed back to the modification of the CAD model.

This paper proposes a novel modeling technology called CADSS (computer-aided design-supporting-simulation). The CADSS model focuses on the functional realization of the product and constructs the relationship between features and parameters in design, modeling, and analysis in order to solve the problem of modeling efficiency in the design and analysis process. The CADSS features can reflect engineering knowledge, design principles, and simulation analysis requirements. Finally, as a typical application of multiphysics simulation, the design of the turbine blade was studied as a meaningful case to illustrate the application of CADSS technology.

\section{Multiphysics Modeling for Simulation}

2.1. Feature-Based Modeling System's Model Representation Method Analysis. The existing feature-based CAD system provides a series of modeling operations through features shown in Figure 1 and defines the relationship with Boolean features for parts or assembling between parts by assembling features. Finally, a product model is generated for simulation or manufacturing.

Based on the process, feature-based modeling technology can be generally defined as three main steps: the setting of reference, justification of dimension and constraint, and preservation of nongeometric information. Therefore, it can be expressed as follows [35]:

$$
F=(g, d, m)=\left(\bigcup_{1}^{u} g_{m}\right) \cup\left(\bigcup_{1}^{v} d_{v}\right)\left(\bigcup_{1}^{k} m_{k}\right),
$$

where $g$ represents geometric information, $d$ represents design intent, and $m$ represents nongeometric information.

For example, to complete a hole feature design, CAD system internal function is CreateCylinder (DP, $\left.R, H, V, O_{i p}\right)$. The input parameters required for the modeling process can be expressed as follows:

$$
P_{m}(f)=\{p(x, y, z), r, h, v(u, v, k)\},
$$

where $P_{m}(f)$ is modeling results using input parameters, $p(x, y, z)$ is datum point, $r$ is radius, $h$ is height, and $v(u, v, k)$ is extruding direction.

The modification and updating of features are relevant to input parameters, i.e., modeling parameters. However, output parameters in the CAD system can be defined as follows:

$$
P_{c}(f)=\left\{g_{c}, d_{c}, m_{c}\right\},
$$

where $g_{c}$ represents cylinder entity, $d_{c}$ represents the design intent of cylinder, and $m_{c}$ represents the cylinder's nongeometry information such as material or color.

According to the above expression, the input parameters and output parameters of the model are inclusive but not identical. Secondly, the definition of design intent is not clear. These parameters may be dimensional such as $r$ in equation (2) or direction constraint such as $v(u, v, k)$ in equation (2). To express a model for simulation requires additional work by engineers to define its semantic information such as $d_{c}$ and $m_{c}$ in equation (3). Nongeometric information about materials and colors is stored in annotations in the model and cannot be updated with the model. These drawbacks undoubtedly affect the quality and efficiency of the CAD model to the subsequent simulation process. Therefore, how to transfer the CAD model and its parameters to the analysis process quickly and accurately needs further exploration. 


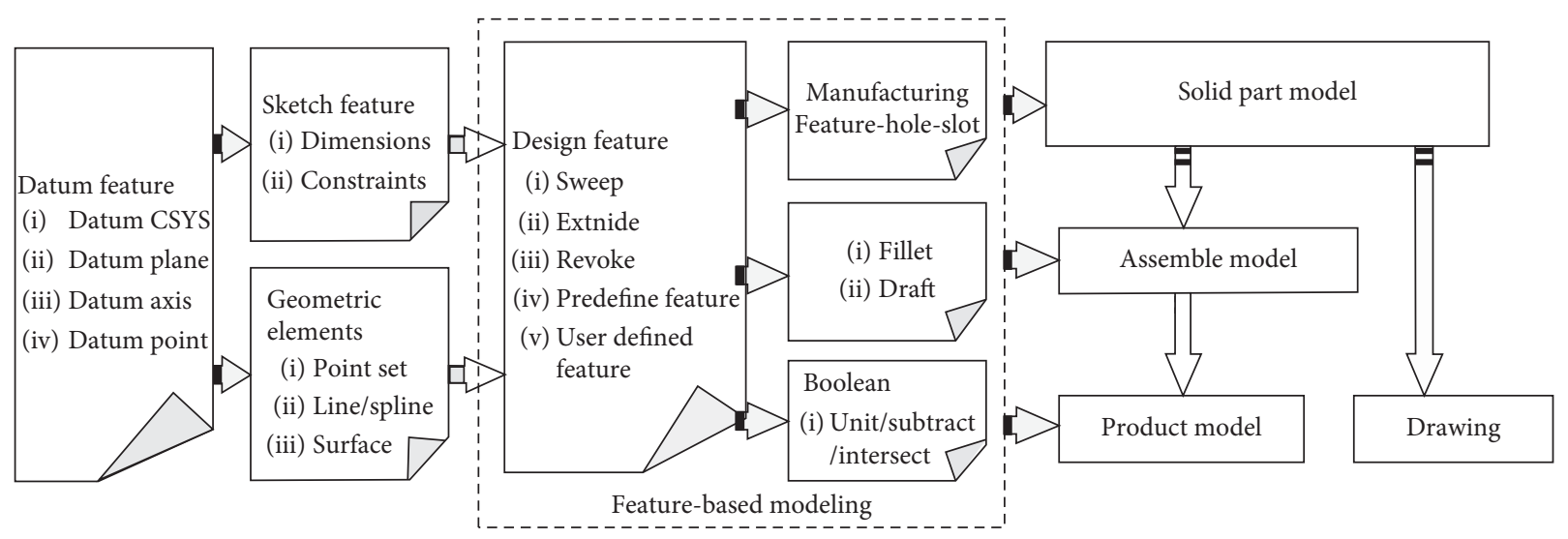

$\Longrightarrow$ Supporting

FIGURE 1: Feature-based CAD system modeling process.

\subsection{Multiphysics Domain Model Representation Method} Analysis. The existing multiphysics domain modeling also encounters the parameter conversion problem mentioned, especially even when it comes to optimization. Though we have adopted a more simplified way for modeling and simulation [36], the use of complex models has more verification functions and industrial application value [37]. The preprocessing, calculation, and postprocessing of simulation still require repeated work for modifying model and calculation conditions even after determining the analysis goals and methods. Furthermore, different methods for describing the same object or using different definitions for the domain can cause that domain model is not adapting to the designanalysis iterative process.

A typical example of computational fluid dynamics and heat transfer analysis for film cooling [38] is shown in Figure 2. Its analysis goal can be defined as follows:

$$
\begin{aligned}
& \eta_{f}=k\left(\frac{X}{\mathrm{MS}}\right)^{\xi}+c, \\
& M=\frac{\rho_{g} v_{g}}{\rho_{c} v_{c}},
\end{aligned}
$$

where $\eta_{f}$ represents film hole cooling efficiency, $k$ is the coefficient of experience related to the location of the hole, $M$ is the blowing ratio, $S$ is the equivalent radius, $\rho_{g} v_{g}$ is gas mass flow, and $\rho_{c} v_{c}$ is cooling air mass flow.

According to the expected cooling efficiency and a certain blowing ratio, the equivalent radius of the desired design can be obtained through equations (4) and (5). When the circular hole is specified in Figure 2, the calculation formula can be given by

$$
\begin{aligned}
S & =\frac{n}{H} \frac{\pi d^{2}}{4}, \\
H & =(n-1) t,
\end{aligned}
$$

where $H$ is the height of one-row hole, $n$ is the number of holes, $d$ is hole radius, and $t$ is spacing of adjacent film hole.

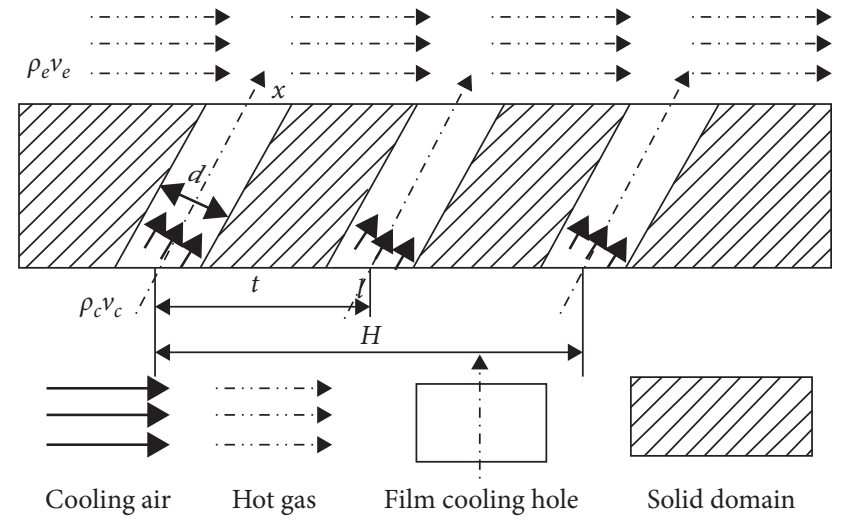

Figure 2: Flat film cooling design diagram.

By extruding, hole operation, and instant hole copy, the geometric entities to be analyzed are initially formed. From the CAD model expression, modeling parameters can be expressed as follows:

$$
P_{m}(f)=\{p(x, y, z), r, h, v(u, v, k), n, t\},
$$

where $p(x, y, z)$ is datum point, $r$ is radius, $h$ is the depth, $v(u, v, k)$ is extruding direction, $n$ is the number of copies, and $t$ is spacing of adjacent film hole.

On the other hand, CFD software needs to construct a complete fluid calculation domain for analysis. The usual practice is to construct a complete inclusion solid to simulate the range of fluid flow and then is to form a fluid domain containing cold air and gas by subtracting the solid portion as shown in Figure 3.

Therefore, in order to achieve multiphysics simulation, simulation parameters are needed as follows:

$$
P_{S}(f)=P_{\text {domain }} \cup P_{\text {boundary }} \cup P_{\text {material }} \cup P_{\text {mesh }} \cup P_{\text {compute }} \cup P_{\text {post }} \text {, }
$$

where $P_{\text {domain }}$ represents computational domain parameters including fluid domain and solid domain, $P_{\text {boundary }}$ represents boundary parameters including inlet, outlet, wall, 


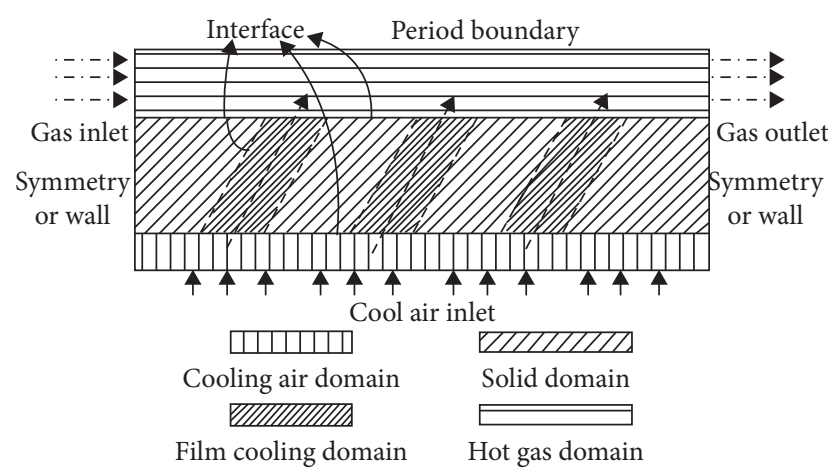

FIGURE 3: Multiphysics model for film cooling flat simulation.

symmetry, opening, interface or interior, and their dependent surface and physics variable. $P_{\text {material }}$ represents material parameters for multiphysics domain such as rigid elastic modulus, fluid thermal conductivity, and specific heat capacity. $P_{\text {mesh }}$ represents parameters of mesh method, mesh size, and mesh control such as tetrahedron, hexahedron, prism, and their appending geometry. $P_{\text {compute }}$ represents parameters of different domain physics model, calculation accuracy, and iteration times. $P_{\text {post }}$ depends on analysis goal and performance evaluation. Specifically, reference geometry and parameters can be made to the illustration of Figure 3.

From the geometrical point of view, the conventional method is to aggregate a number of geometric entities with geometric shapes and forms, and there is no integration of the properties of the relevant features. The computational domain geometry's modification requires geometric adjustment from the CAD or even the initial design parameters. Boolean operation usually causes errors or unexpected results due to the complexity of the model. The designer needs to perform geometric inspection and repair even sewing.

Table 1 shows the parameter difference for CAD and simulation. The setting of the boundary conditions and the division of the mesh in the preprocessing of the numerical simulation are heavily dependent on the geometry of the computational domain as equation (9) defines. However, cooling air and gas domain is a negative entity when modeling in the CAD system shown in Figure 2. They do not exist or are not preserved during the modeling process. Besides, these fluid regions in Figure 3 should have been molded for meshing, setting boundaries, and physics model for numerical simulation.

According to the above analysis, the simulation parameters are dependent on the geometry of the multiphysics model. Therefore, it is necessary to construct a multiphysics model and adopt a unified parameter expression method for the same object in the modeling stage, which is necessary to solve the problems of model adaptation and efficiency for multiphysics simulation or MDO.

2.3. Multiphysics Domain Model Architecture. Through the analysis of the model representation method, the relationship between multiphysics domain model parameters (MMDP) and modeling parameters (MP), design parameters (DP), simulation parameters (SP), and optimization parameters (OP) was summarized, as shown in Figure 4.

Updating domain model depends on its domain parameters. Through product requirements, the rational design model (DM) was designed using physical parameters determined by multiple disciplines' knowledge and was evaluated through performance parameters. Then, a CAD model (MM) is generated, and optimization objective function and constraint function can be generated as an optimization model (OP). Moreover, the multiphysics domain model (MDM) is carried out and should be relevant to the designed CAD model. The feature parameters are needed to be inherited. When it comes to multiphysics domain numerical simulation, simulation model (SM) generation is generally divided into three stages: preprocessing, calculation, and postprocessing. Three stage parameters are associated with multiphysics domain geometric and are summarized in three aspects as follows:

(1) Preprocessing: multianalysis domain model, meshing (boundary layer mesh, local refinement, and mesh match), material properties (elastic modulus), boundary conditions (inlet and outlet, wall, symmetry, and periodic boundary), displacement constraints, and boundary conditions

(2) Calculation: determination of the analytical analysis model (fluid domain calculation model, residual, and number of iterations)

(3) Posttreatment: temperature cloud map, flow rate map, equivalent stress, etc.

Therefore, when building a CAD model, the designer should understand the changes in the foreseeable geometric model and make the geometric model robust enough to cope with the changes. Besides, according to the numerical simulation evaluation results of the physical model, the analyst should also be able to modify the geometric model quickly. Through multiphysics domain model architecture, there are still works to achieve.

(1) Implement requirement decomposition and functional decomposition [37] for appropriate domain parameter extraction

(2) Modeling method to achieve different domain knowledge storing and updating in one model

(3) Achieve multiphysics domain parameters transferring to each other

\section{Computer-Aided Design-Supporting- Simulation Technology}

3.1. Proposed Method. The product's function refers to the ability to meet users' requirements while users need to form a demand space. Each requirement corresponds to the function. The definition and description of each function should not only meet the specific user requirements, but also reflect the technical principle of the function realization. Although the functional definitions of products in different fields are different, the functional decomposition performed 
TABLE 1: Parameters difference for CAD and simulation.

\begin{tabular}{|c|c|c|}
\hline Application type & Input parameters & Output parameters \\
\hline Modeling in CAD & $\begin{array}{l}\text { Datum } \\
\text { (i) Datum CSYS, datum plane, datum axis, datum point } \\
\text { Dimension } \\
\text { (ii) Length, angle, number }\end{array}$ & Geometric entity \\
\hline Modeling for simulation & $\begin{array}{l}\text { Domain body } \\
\text { (i) Solid domain, fluid domain } \\
\text { Mesh } \\
\text { (ii) Boundary layer mesh, local refinement, and mesh match } \\
\text { Boundary condition } \\
\text { (iii) Inlet, outlet, wall, symmetrical plane, periodic boundary } \\
\text { Material } \\
\text { Solver setting }\end{array}$ & $\begin{array}{l}\text { Simulation results } \\
\text { (i) Temperature and pressure fields files } \\
\text { (ii) Maximum stress } \\
\text { (iii) Objective functions' values }\end{array}$ \\
\hline
\end{tabular}

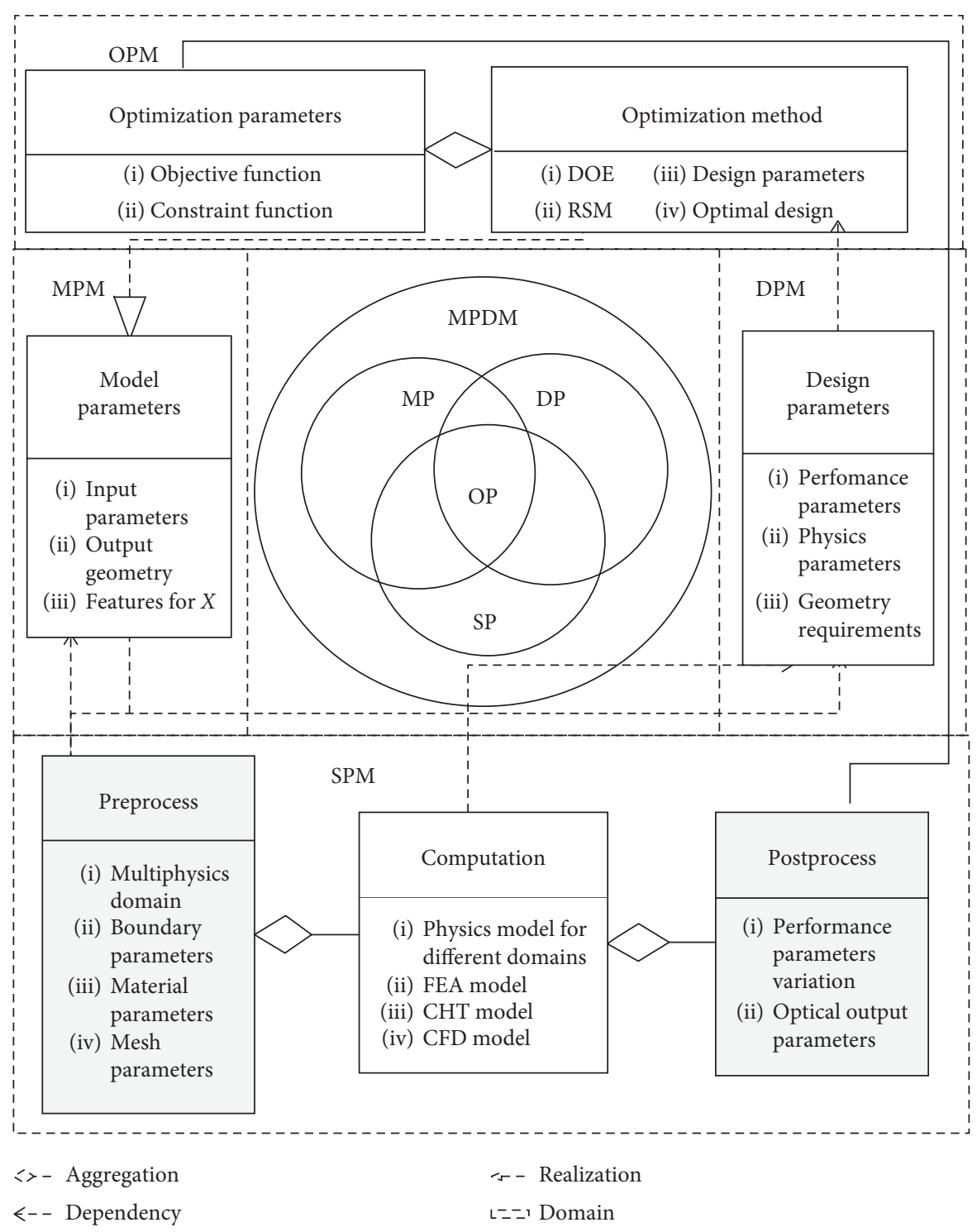

FIGURE 4: Structure of multiphysics domain model architecture. 
under the CADSS framework is based on the knowledge space as a carrier to consider the subsequent verification process from top to bottom in the process of modeling. As shown in Figure 5, CADSS system contains three aspects including requirement space, domain knowledge, and software technology as follows:

$$
S_{\text {CADSS }}=\left(S_{R}, S_{K}, S_{T}\right) .
$$

A collection of all requirements can be expressed as follows:

$$
S_{r}=\left\{r_{i} \mid i=1,2, \ldots, n\right\} .
$$

Generally, each requirement corresponds to a function or a set of features, and each function may also correspond to a combination of multiple features. Domain knowledge includes different analysis domains and mathematical models. Firstly, analyzing and decomposing product functions are required to embed the domain knowledge into functional features. And then using relevant software technologies (such as specific geometric algorithms, parametric modeling methods, and numerical simulation methods) can achieve simulation requirements. The next section elaborates on the use of functional features for CADSS technology.

3.2. Multiphysics Model Representation in CADSS. CADSS aims to establish a functional space for product design. Therefore, parameter relationship between design, modeling, and simulation can be bridged. According to Figure 5, all functional elements can be expressed as follows:

$$
\begin{aligned}
F & =\left\{f_{i} \mid i=1,2, \ldots, n\right\}, \\
f_{i} & =S_{f}\left(r_{i}, k, t\right),
\end{aligned}
$$

where $S_{f}$ represents the design process, requirements $r_{i} \in S(R)$, domain knowledge $k \in S_{k}$, and software technology $t \in S_{T}$.

The realization of functional features after decomposition by means of parameter decomposition is as follows:

$$
P_{\text {CADSS }}(f)=P_{d}(f) \cup P_{m}(f) \cup P_{s}(f),
$$

where $P_{d}(f)$ is defined in equation (2), $P_{m}(f)$ is defined in equation (3), and $P_{s}(f)$ is defined in equation (9).

The way defined by the above parameters can be used to build the connection between design, modeling, and simulation, thus expanding the scope of application of the feature. Through the domain knowledge, the mapping relationship of the three types of parameters can be realized as follows:

$$
\left\{\begin{array}{l}
P_{m}(f)=K_{1}\left(P_{d}(f)\right), P_{d}(f)=K_{1}^{-1}\left(P_{m}(f)\right), \\
P_{s}(f)=K_{2}\left(P_{m}(f)\right), P_{m}(f)=K_{2}^{-1}\left(P_{s}(f)\right), \\
P_{s}(f)=K_{2}\left(K_{1}\left(P_{d}(f)\right)\right), P_{d}(f)=K_{1}^{-1}\left(K_{2}^{-1}\left(P_{s}(f)\right)\right) .
\end{array}\right.
$$

In general, the meaning of $K$ can be expressed by the following triplet:

$$
(X, K, Y)
$$

where $X=\left(x_{1}, x_{2}, \ldots, x_{n}\right), Y=\left(y_{1}, y_{1}, \ldots, y_{n}\right)$, and $X$ is a set of parameters of the $P_{\text {CADSS }}(f)$ space. $K$ represents inference conditions, reasoning processes, or geometric algorithms.

According to the definition of $K$, design reasoning can be divided into parameter calculation, rules reasoning, chart query, and empirical solution.

3.2.1. Parameter Calculation. A set of parameters is known and is a scalar parameter, and the unknown parameters can be calculated by solving the following equations:

$$
\begin{aligned}
& Y=E(X), \\
& E=\left(e_{1}\left(X_{1}\right), e_{2}\left(X_{2}\right), K \ldots, e_{n}\left(X_{n}\right)\right) .
\end{aligned}
$$

For example, via equations (4)-(7), the number, spacing, and radius of holes can be calculated by the estimated cooling efficiency of the heat transfer design.

3.2.2. Rules Reasoning. Representation of composite features by logical expressions is shown in the following equation:

$$
\left(X, L_{c}, Y\right) .
$$

For instance, feature-based shape inference rules can be expressed as follows:

$$
\left(f_{\text {gas }}, f_{\text {cool }}=f_{\text {fluid }} Y\right),
$$

where $f_{\text {gas }}$ represents gas domain features, $f_{\text {cool }}$ represents cooling air domain features, $f_{\text {fluid }}$ represents fluid domain features, and $Y$ represents interface or Boolean operation depending on domain knowledge.

3.2.3. Chart Query and Empirical Solution. Under this condition, $K$ represents experiment data or experience. Therefore, given $X$, with expertise and experience $K, Y$ can be determined by $C_{Y}$ in an empirical chart:

$$
Y=C_{Y} .
$$

The conversion of the above design parameters, modeling parameters, and simulation parameters is carried out by different engineering personnel. In order to ensure the accurate and efficient conversion of the above mapping relationship, the knowledge of the above domain needs to be solidified by the corresponding software technology, which can be expressed as follows:

$$
T_{\text {CADSS }}=T\left(K_{f_{1}} \cup K_{f_{1}} \cup, \ldots, \cup K_{f_{1}}\right) .
$$




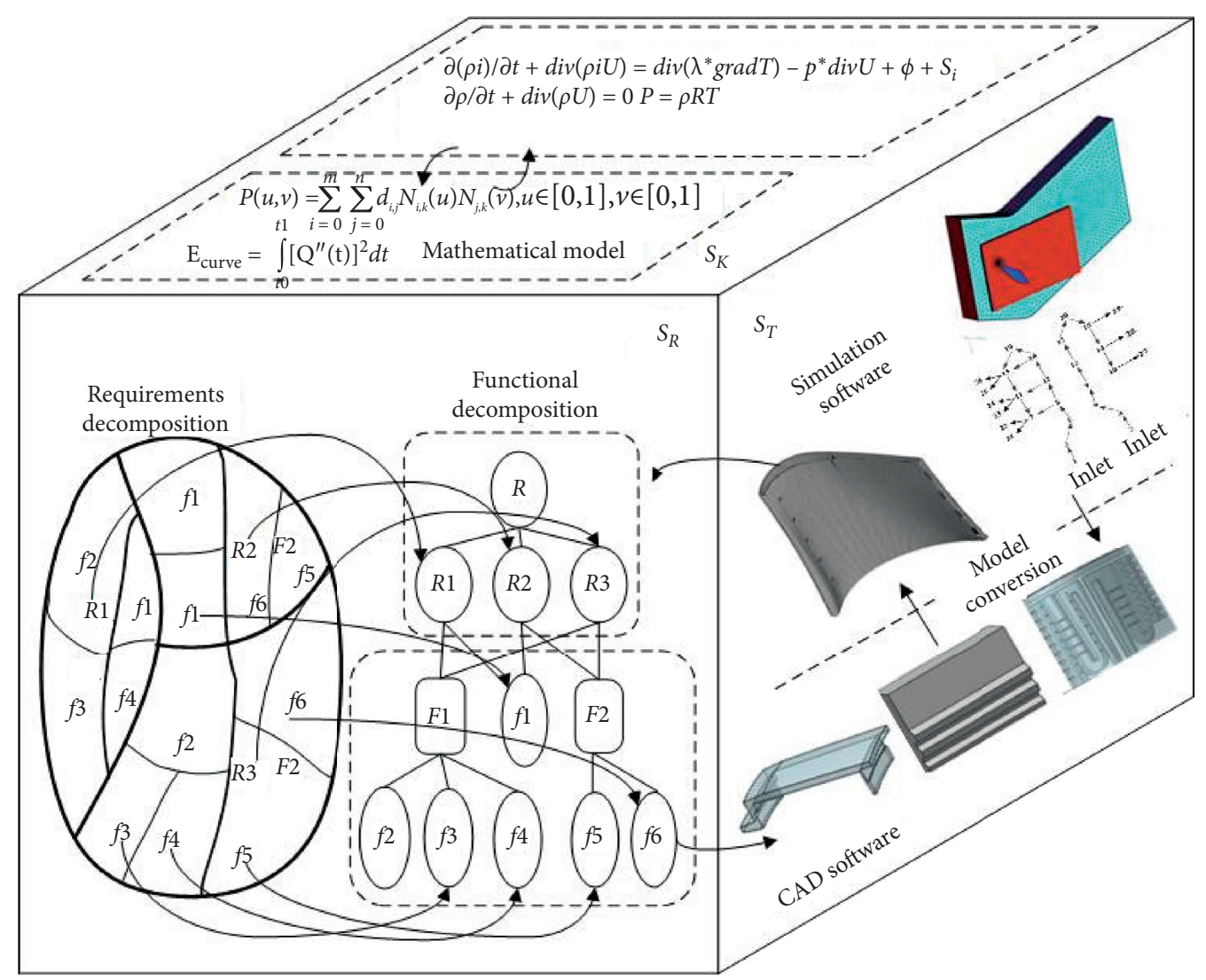

FIGURE 5: CADSS framework.

It can be realized through programming and related algorithms through CAD software, CAE analysis software, and MDO software. The next section will show how CADSS technology is conducted in a typical design case with multiple physical modeling, simulation, and optimization.

The design of the turbine blade is chosen to be the case study in this paper because it shows how the product requirements and function are decomposed to modeling features. It demonstrates how the concept of MMDP is transferred among DM, MM, SM, and OM and how they are embedded into a CAD model.

\subsection{CADSS Implementation for Turbine Blade}

3.3.1. Requirement Space and Functional Decomposition of Turbine Blades via CADSS Technology. The working environment of turbine blades is becoming increasingly harsh with the continuous increment of turbine inlet temperature. And the coupling effects between aerodynamics, heat transfer, and strength are becoming more and more significant for turbine blade design. It is urgent to start research on multidisciplinary design optimization methods for turbine blades compared with multiobjective optimization of discrete film hole [39]. The design optimization flowchart is shown in Figure 6.

Firstly, according to the overall design requirements of the original designed turbine blade, the initial design variables and the objective functions are determined. Then, a preliminary DOE is performed to generate several design points for simulations shown in the right of Figure 6, which is the general process for multifield coupling analysis [40]. Secondly, according to the various design points by the DOE design, the geometric model was reconstructed in the CAD software according to design variables. On the one hand, the fluid domain and solid domain geometric models are constructed for CHT simulation, and then the fluid mesh is generated based on the solid and fluid models in the meshing software. The fluid grid and solid grid are numerically solved in the computational fluid dynamics solver, and the temperature and pressure fields of the turbine blade are predicted to determine whether the objective functions are met. On the other hand, in the finite element calculation, based on the solid model of the turbine blade, the temperature field and pressure field data need to be input to the finite element analysis as boundary conditions for strength check to further determine whether the maximum stress of the blade meets the objective functions. Thirdly, the construction and improvement of the approximation model is generated with DOE results. Finally, a multidisciplinary and multiobjective optimization algorithm is used to find the optimal turbine blade model for the current design variables and objective functions.

Turbine blade overall design requirements are based on both strength and longevity conditions, using cooling air flow as less as possible to achieve higher cooling efficiency.

The design requirements can be expressed mathematically as equations (23)-(25): 


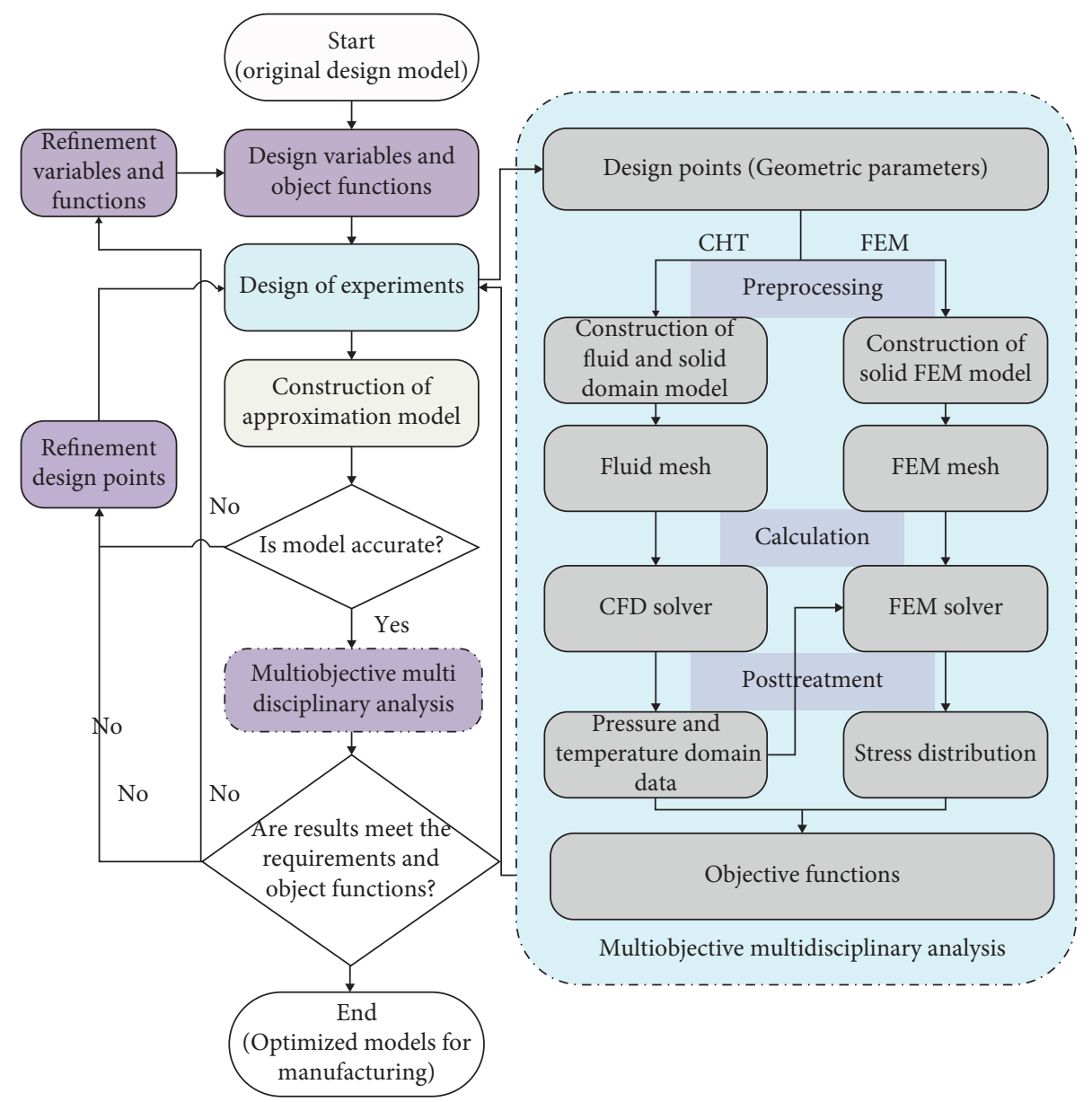

FIGURE 6: Design optimization flowchart.

$$
\eta_{1}=\frac{T_{g, r}-T_{a w}}{T_{g, r}-T_{c}}
$$

where $\eta_{1}$ is cooling efficiency, $T_{g, r}$ is gas recovery temperature, $T_{a w}$ is adiabatic wall temperature, and $T_{c}$ is cooling air temperature.

$$
\begin{aligned}
\eta_{2} & =\frac{T_{\max }}{T_{\text {ref }}} \frac{A_{t b}}{A_{b}} \frac{1}{1-\chi}, \\
\chi & =\frac{T_{b}}{T_{*}},
\end{aligned}
$$

where $T_{\text {ref }}$ is the reference temperature, $T_{\max }$ is the maximum temperature on the blade surface, $A_{b}$ is the blade surface area. $A_{t b}$ is the area where the temperature is higher than $T_{b} \cdot \chi$ is temperature coefficient, and $T_{*}$ is gas total temperature.

$$
\sigma_{\max } \leq 0.8 * \sigma_{0.2}^{T}
$$

where $\sigma_{0.2}^{T}$ represents $0.2 \%$ yield strength of the material at normal blade operating temperatures. $\eta_{1}$ reflects the cooling efficiency of the blade cooling structure. $\eta_{2}$ reflects the worstcase temperature field during blade operation, which is related to blade lifetime. $\sigma_{\max }$ reflects the maximum strength experienced by the blade.
According to requirements, the composite cooling structure is adopted for reducing weight and enhancing cooling air efficiency. As shown in Figure 7, according to a different subject domain, the functional analysis and decomposition of the turbine blades are carried out to obtain a functional decomposition tree. The functional structure decomposition tree is organized in the form of functional features, and the top-down design is used to perform functional features management. Finally, the turbine blade 3D CAD model and multiphysics simulation preprocessing model are generated.

After the requirement decomposition, the next step is to customize the blade model according to the requirement decomposition design scheme to quickly complete the generation of the calculation domain. The traditional design process does not include the modeling history and only knows the modeling results, and the results are all nonparametric solid model; fluid domain generation contains a lot of engineering design experience and frequent manual interaction, and the modeling process is irregular and does not support the rapid modification and serial design of the blade model. The numerical analysis process is an iterative process of modifying and reanalysing the blade model according to the calculation results and constructing and adjusting a nonparametric solid model that lacks a modeling history, repeated work, and complicated adjustment process, and the quality of the model cannot be guaranteed. 


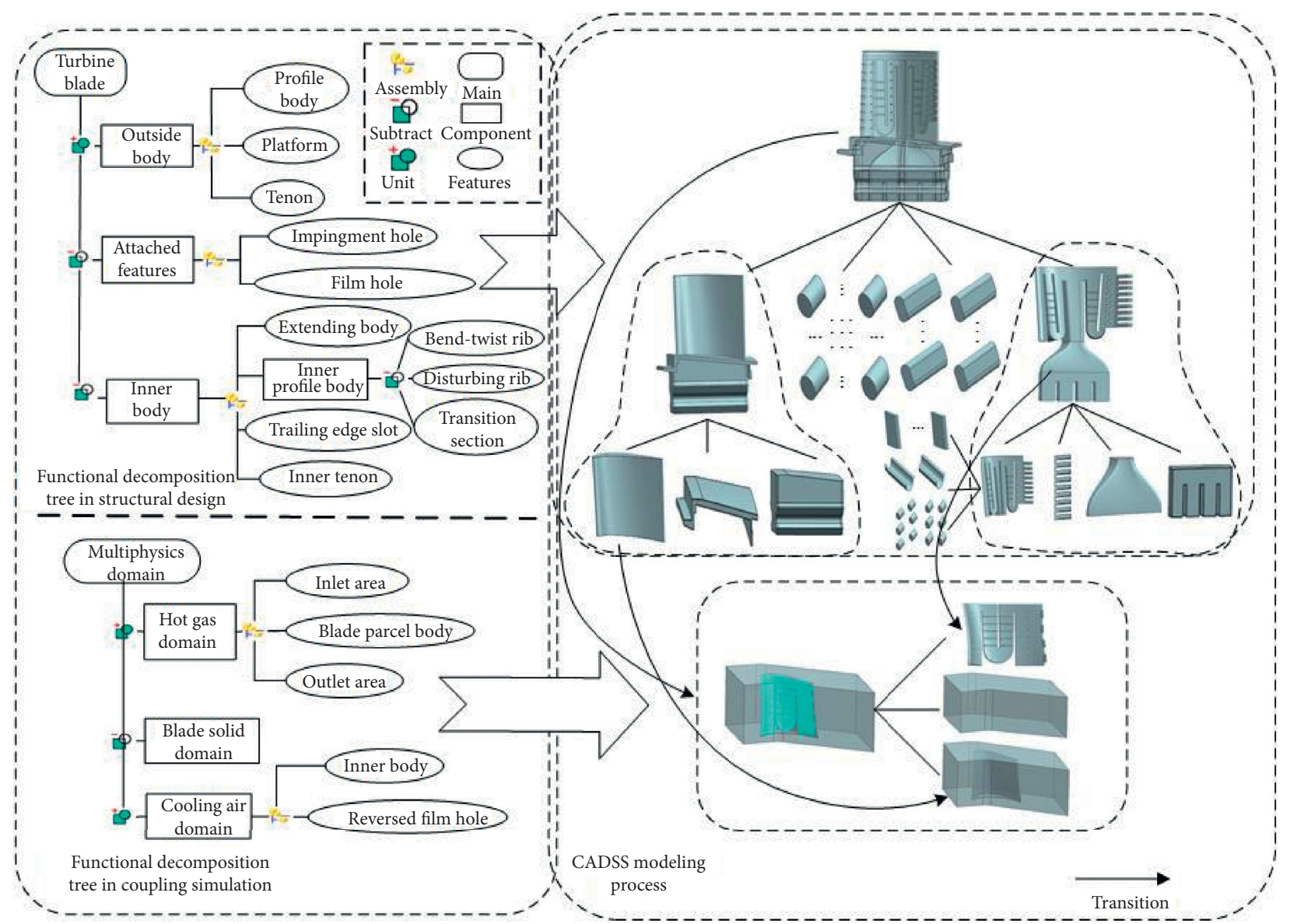

FIgURE 7: Functional decomposition of turbine blades via CADSS technology.

In addition, the above modeling method only considers the construction of the geometric model of the calculation domain and does not analyze the entire process of gas-heat coupling numerical simulation and considers the requirements of subsequent analysis for modeling. In fact, in the postprocessing stage, in order to obtain the temperature distribution, pressure distribution of the critical position of the blade, and the cooling air flow, speed, and pressure of the cooling structure, it is necessary to extract the geometric elements in the calculation domain model and then observe and analyze the calculation results, and the location and generation of complex geometric features in the analysis software are difficult, require a large amount of work, and cannot guarantee accuracy.

According to the above analysis, the requirements of the gas-heat coupling numerical simulation process for the computational domain modeling are as follows:

(1) A general method for the rapid construction of the turbine blade inflow cold air region

(2) Extraction of analysis information such as the positioning and generation of key geometric features

(3) The generation of outflow gas domain should be compatible with the blade model and can be flexibly adjusted
Among them, (1) need to design methods to avoid the need to rely on experience in the process of generating cold air regions; (2) need to extract methods for key geometric features according to the requirements of the postprocessing stage; (3) mean that according to the boundary of gas-heat coupling analysis. The conditions construct a method of generating outflow gas domains that can adapt to different blade shapes and cooling structures.

\subsubsection{Model Design with CADSS}

(1) Different Analysis Domain Model Generation Scheme. According to the above decomposition and design scheme, turbine cooling blades were divided into typical features as shown in the following equation:

$$
F_{m}=\left\{f_{i}\left(P_{\text {CADSS }}\right) \mid i=1,2, \ldots, 13\right\},
$$

where $f_{1}, f_{2}, \ldots, f_{13}$ is shown in Figure 8 .

The basic feature set of any turbine cooling blade was $F_{m}^{\prime}$, $F_{m}^{\prime} \subseteq F_{m}$. Blade model is represented as follows:

$$
M_{b}=\left(M_{o}, M_{i}, f_{12}, f_{13}, e_{b}\right)
$$


where $e_{b}=\left\{e_{b}^{j} \mid j=1,2,3\right\}$, when subtraction is operated, $e_{b}$ equals zero, and when uniting, $e_{b}$ equals one.

Blade inner body is represented as follows:

$$
M_{i}=\left(f_{6}, f_{7}, f_{8}, f_{9}, f_{10}, f_{11}, f_{5}, f_{4}, e_{i}\right) .
$$

Blade outside the body can be represented as follows:

$$
M_{o}=\left(f_{1}, f_{2}, f_{3}, e_{o}\right) .
$$

Take $f_{13}$ for example, its design parameters depend on $\eta_{f}$, relevance variables in equation (4), and estimated gas temperature and cooling air temperature. Tables 1 and 2 show the parameters of the blade designed in this paper. Through equation (6), the modeling parameters are calculated. In the CAD system, $f_{13}^{-1}$, the film hole fluid domain corresponding to $f_{13}$, can be generated and its simulation parameters such as mesh size control and inflating method can be assigned to $f_{13}^{-1}$.

Therefore, it is possible to design a turbine blade computational domain modeling solution for gas-heat coupling (Figure 9), generate the inner shape and cooling structure of the blade body according to the inner section line and modeling parameters, and then automatically locate the outer cooling features to achieve the cold air domain. The rapid modeling meets the requirements (1); according to the profile section line and blade modeling parameters, the blade shape is created, and then the adaptive blade periodic boundary and the blade inclusion are created, and then according to the blade periodic boundary and the wall boundary as well as the direction and length of the entrance and exit, adaptive cutting inclusions for rapidly modeling gas field is generated, which meets the requirements (3); in the process of modeling the cold gas field and the gas field, according to the relevant modeling parameters, the extraction of geometric features and the preservation of nongeometric information meet the requirements (3).

(2) Computational Domain Automatic Generation Algorithm. The calculation model of the turbine blade in multiphysics numerical simulation mainly includes the fluid calculation domain and the solid calculation domain. According to the flow field characteristics and the mesh division strategy, the fluid calculation domain can be divided into the gas domain and the cold gas domain.

Specifically as shown in Figure 10, according to the flow path entity $M_{b}$ created by the existing blade solid model $M_{b}$ and the blade flow path information, the calculation domain model is constructed by artificial interaction. The fluid calculation domain $M_{f}$ is formed by subtracting between $M_{p}$ and $M_{b}$. With the method of segmentation and combination, a gas domain $M_{g}$ and a cooling air domain $M_{c}$ were divided by the fluid domain.

During the modeling of fluid domain, the simulation parameters can be generated. The boundary attached on $M_{f}$ includes a gas inlet, gas outlet, cooling air inlet, period surface, and wall. Mesh control parameters can also be embedded into the fluid entity. The interface generated by
$M_{f}$ and $M_{b}$ needs to assign inflation mesh control for the requirement of y-plus depending on the physics model. Material parameters are appended to the target entity for simulation. Blade surface and fluid surface for postprocess evaluating performance parameters such as temperature and pressure are marked by name to be identified in the postprocess module.

The creation of the gas domain model needs to adapt to different blade profiles, and it is necessary to combine the common geometrical characteristics of the blades with the characteristics of the outflow gas domain. The midblade arc is not only an important parameter for aerodynamic shape design but also an important design reference for the inner shape of the blade. It can reflect the bending and torsional changes of the blade in the flow channel, which meets the conditions of periodic boundary selection. The main difficulties are the automatic matching of periodic boundaries and the adaptive cutting of blade inclusions.

Adaptive pipeline intersection algorithm is shown in Figure 11. First of all, in the past, the method of creating intersection arcs in the pipeline intersection was mainly based on empirical data to obtain the pipeline radius, and the generation of periodic boundaries needs to adapt to different blade shapes. The pipeline radius data cannot automatically match the blade shape, so the improved adaptive pipeline is used. The intersection algorithm can solve this problem; secondly, due to the intersection of the pipeline and the partial encryption and streamlining of the blade cross section line, a variety of curves shown in Figure 11 will be generated, and it is necessary to use an automatic recognition algorithm to identify the middle arc from the curve of Figure 11.

Step 1: traverse the blade profile section line, according to the height and curve type, automatically match each layer of the leaf back curve $L_{k}$ and the leaf pot curve $L_{k}$ (the number $k$ of section line layers), and discretize the $L_{k}$ and $L_{k}^{\prime}$ according to the parameter method of equal arc length to obtain the layer respectively point set.

Step 2: calculate the distance between the points in the two points separately $\left|\overrightarrow{P_{i} S_{j}}\right|$. The maximum wall thickness of the airfoil is obtained by trial calculation $C_{\max }^{\prime} \longleftarrow \max \left\{\min \left(D_{i}\right) \mid i=0,1,2, \ldots, n-1\right\}$. The diameter of the pipeline in the pipeline intersection is $0.75 C_{\max }^{\prime}$, which is the radius of the pipeline of each layer of cross section line.

Step 3: with the $L_{k}$ and $L_{k}^{\prime}$ starting point as the center of the circle, create a circle with a diameter $d_{k}$ in the normal plane of the starting point of the curve, and as the guide line, and after sweeping, the two pipes intersect to obtain $C_{P}$ and $C_{P}^{\prime}$, and project onto the plane of the section line of this layer to obtain the midarc $C_{k}$.

Adaptive generation method of gas domain is shown in Figure 12. The blade model obtained by improving the pipeline intersection algorithm is processed by the periodic boundary automatic matching method and the outflow gas 


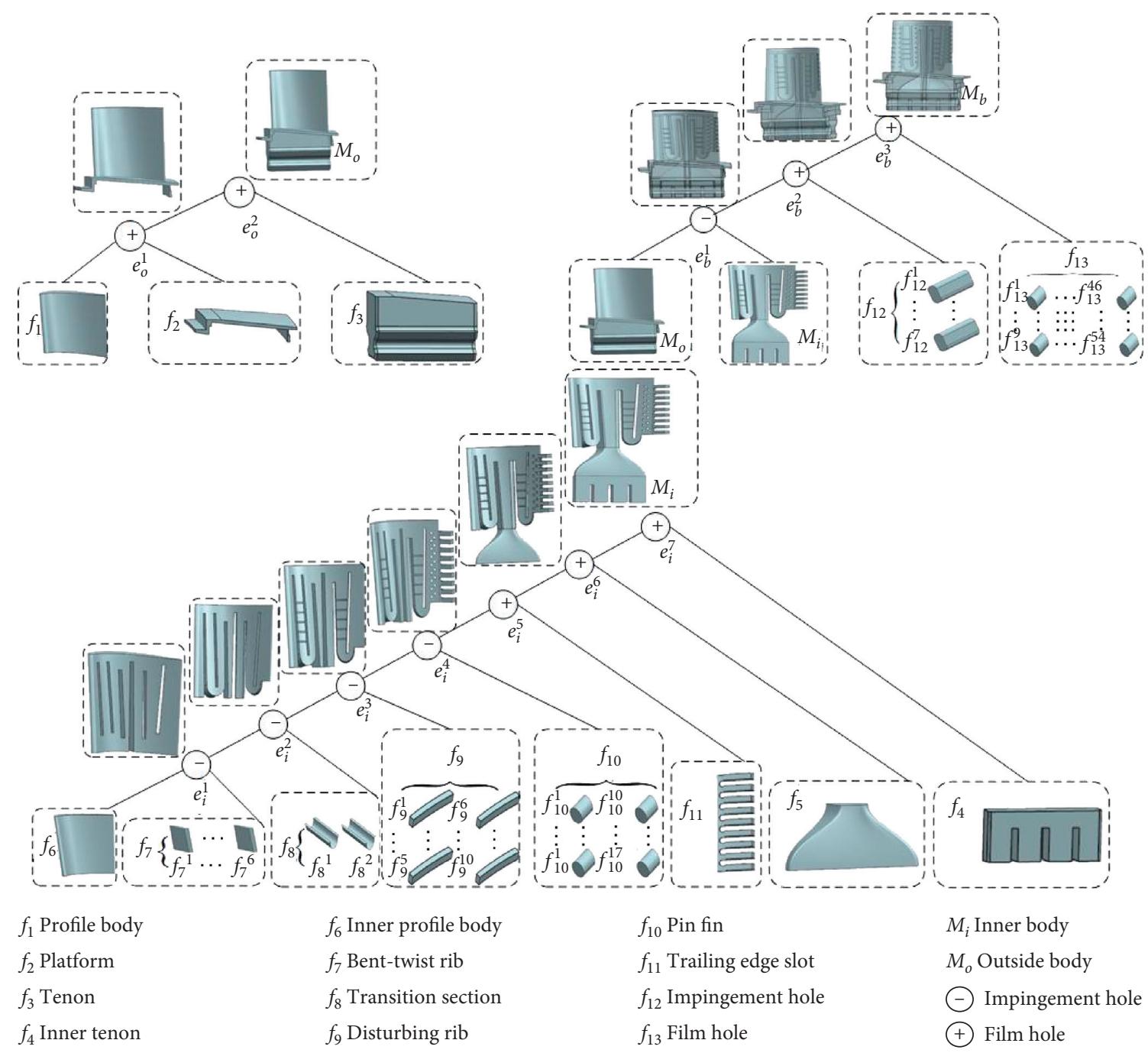

FIgURE 8: CADSS feature representation in CADSS.

TABLE 2: Tags in CAD software for simulation.

\begin{tabular}{|c|c|c|}
\hline Tag and type & Attribute & Boundary condition \\
\hline $\mathrm{NS}_{-} I_{1}, \mathrm{NS}_{-} I_{2}, \ldots, \mathrm{NS}_{-} I_{n}$ & Inlet & Velocity or pressure inlet \\
\hline NS_O $O_{1}$, NS $\_O_{2}, \ldots$, NS_O $O_{n}$ & Outlet & Velocity or pressure inlet \\
\hline NS $-W_{1}, N_{-} W_{2}, \ldots, N_{-} W_{n}$ & Wall & No-slip wall \\
\hline $\mathrm{NS}_{-} S_{1}, \mathrm{NS}_{-} S_{2}, \ldots, \mathrm{NS}_{-} S_{n}$ & Symmetrical plane & Symmetry \\
\hline NS_ $P_{1}$, NS_$_{-} P_{2}, \ldots$, NS $_{-} P_{n}$ & Periodic boundary & Periodic surface \\
\hline NS $\_D_{1}$, NS $\_D_{2}, \ldots$, NS $\_D_{n}$ & Domain or subdomain & Fluid or solid domain \\
\hline
\end{tabular}

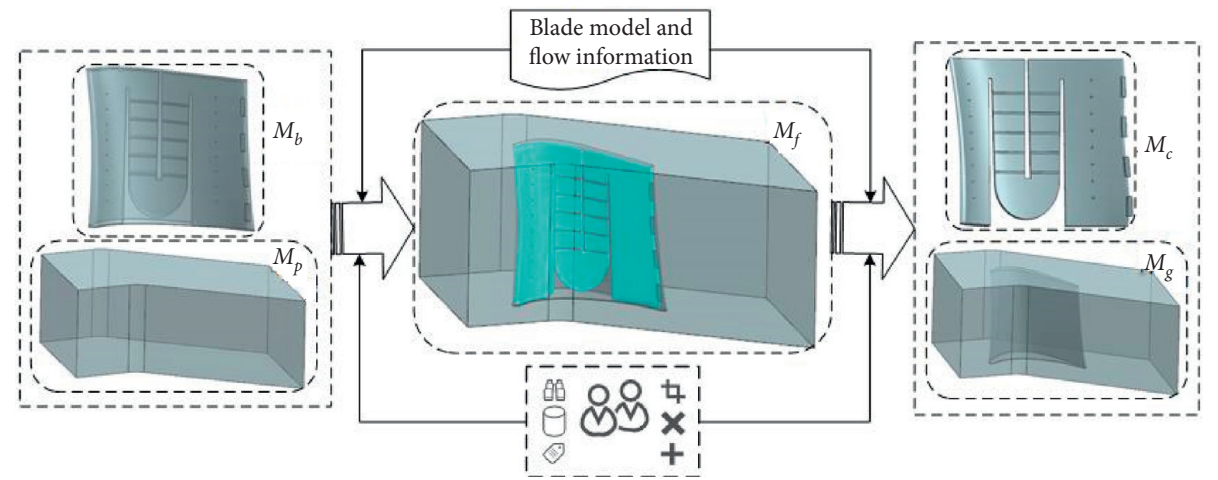

FIGURE 9: Multiphysics numerical simulation model. 


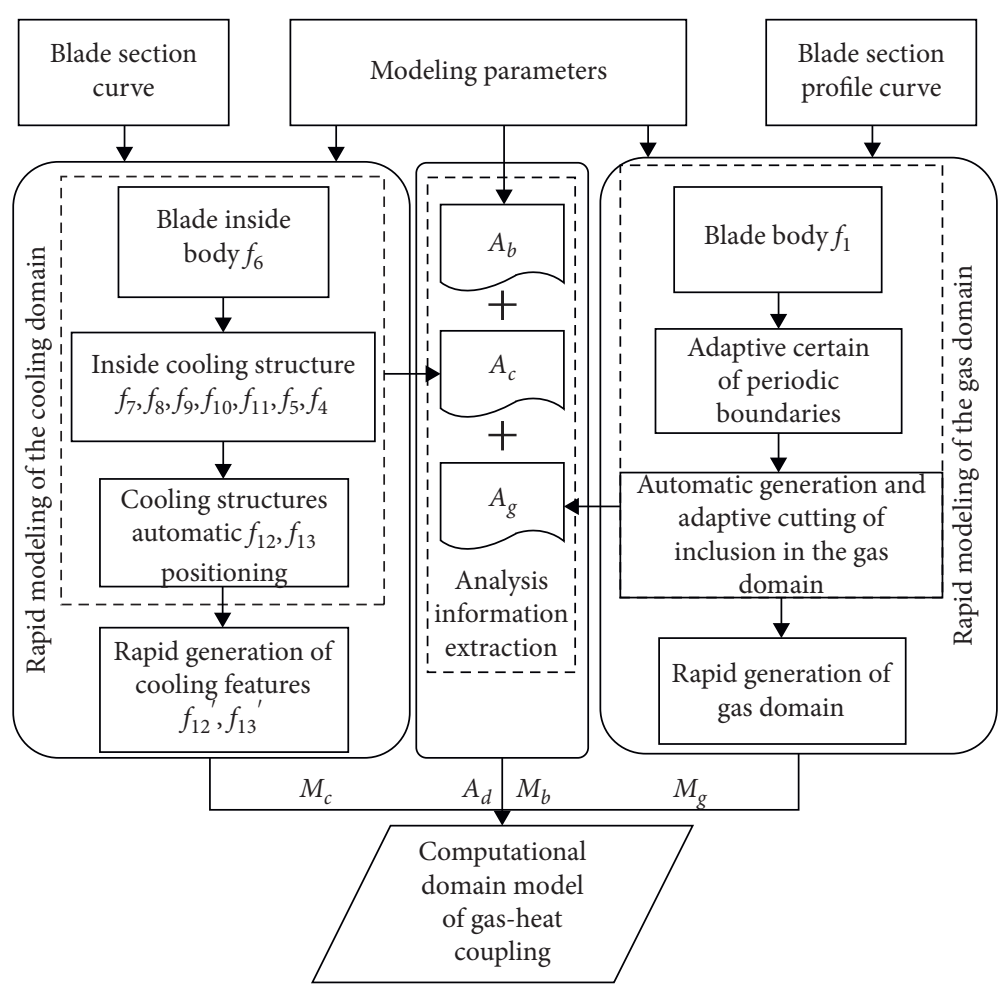

FIGURE 10: Conjugated heat transfer simulation-oriented turbine blade computation domain modeling scheme.
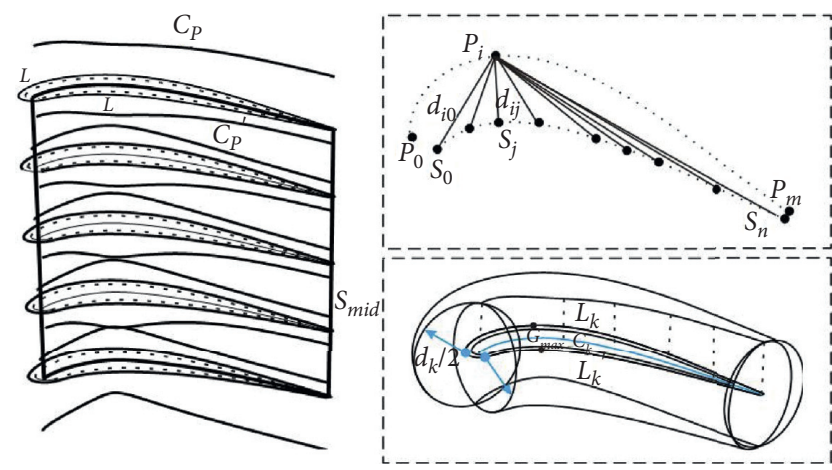

FIgURE 11: Adaptive pipeline intersection for turbine blade.

domain adaptive cropping algorithm, which can adaptively generate the gas domain.

Step 1: according to the height and curve type, filter the pipes in Figure 7 to find the intersection curve $C_{P}$ and $C_{p}^{\prime}$, and group the remaining outer section line, inner section line, and middle arc line according to height, query the minimum bounding box of each group of section line, and compare the smallest surrounding. The bounding box can be divided into outer section line, inner section line, and middle arc line from inside to inside in sequence.

Step 2: according to the grouped midarc lines, loft them in order of height to get the midarc surface and extend the midarc surface $S_{m}^{\prime}$ and $S_{m}^{\prime \prime}$. According to the number of blades on the turbine, the midarc surface rotation angle is obtained.
Step 3: simultaneously calculate the leaf pot, the backside periodic boundary, and the minimum bounding box of the leaf, and create a leaf inclusion $E_{\text {cubic }}$, the minimum enclosing box $B_{c}\left(B_{\text {blade }} \subset B_{c}\right)$, and within the area formed by the backside of the pot, to ensure rotation curved surface and ability to cut inclusions,

Step 4: continue to cut according to the wall boundary; input the inlet and outlet gas angles $\alpha_{1}$ and $\alpha_{2}$, lengths $l_{1}$ and $l_{2}$, stretch and wrap the inlet and outlet surface to generate the blade flow channel entity, and minus the blade entity and the cooling air domain to obtain the outflow gas domain part $M_{g}$.

Through the adaptive pipeline intersection Algorithm 1, the arc in the blade can adapt to the changes of different blades. The periodic boundary automatic matching method and the outflow gas domain adaptive cropping Algorithm 2 both solve the problem of outflow gas domain and blade model adaptation. The flexibility of its modeling requirements has been met through Algorithms 1 and 2 .

(3) Generation of Analysis Domain Model for Simulation and Optimization. The modeling part and algorithm described above can be realized by building a dedicated GUI for analysis-oriented CAD models. For example, in Siemens NX ${ }^{\circledR}$, engineers can use Product Template Studio or Block UI Styler, depending on the complexity of the current product and the user's skill set. If users want to use Block UI Styler, they need to use the application programming interface for programming. Such design may be function- 
Inputs: blade section line $L_{k}$ and $L_{k}^{\prime}$

Outputs: midcurve $C_{k}$

(1) $k \longleftarrow 0$, initialize and traverse the blade profile section line $L_{k}$ and $L_{k}^{\prime}$

(2) while $H_{L_{k}} \longleftarrow h$ do

(3) Discretize the $L_{k}$ and $L_{k}^{\prime}$ according to the parameter method of equal arc length to obtain the layer respectively point sets $P=\left\{P_{i}(x, y, z) \mid i=0,1,2, \ldots, n-2, n-1\right\}, S=\left\{S_{j}(x, y, z) \mid j=0,1,2, \ldots, n-2, n-1\right\}$.

(4) for all point sets $P$ and $S$

(5) Calculate the distance between the points in the two points separately $\left|\overrightarrow{P_{i} S_{j}}\right|$

(6) $C_{\max }^{\prime} \longleftarrow \max \left\{\min \left(D_{i}\right) \mid i=0,1,2, \ldots, n-1\right\}$

(7) $d_{k} \longleftarrow 0.75 * C_{\max }^{\prime}$

(8) Create a circle with a diameter $d_{k}$ in the normal plane of the starting point of the curve, and as the guide line, and after sweeping, the two pipes intersect to obtain $C_{P}$ and $C_{P}^{\prime}$ and project onto the plane of the section line of this layer to obtain the midarc $C_{k}$

(9) end for

(10) $k \longleftarrow k+1$

(11) end while

(12) return $C_{k}$

Algorithm 1: Adaptive pipeline intersection algorithm.

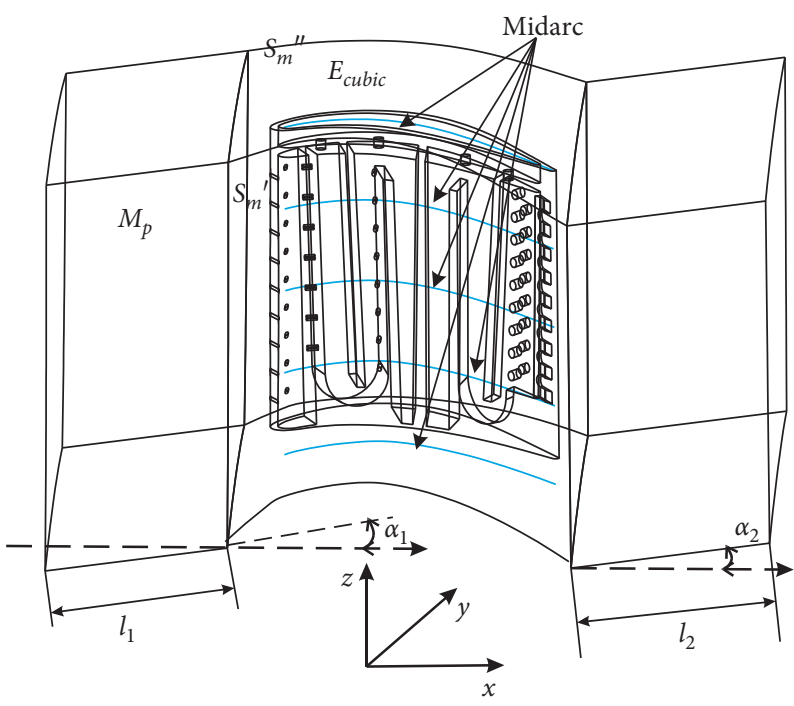

FIgURE 12: Adaptive generation method of gas domain.

centric in the future because users do not need to rebuild CAD models, but provide some functional parameters to automatically generate as shown in Figures 8 and 9. Beneficially, the flow space can be easily updated subject to design changes. Tags with attributes, similar to named selections, are assigned to the fluid geometrical faces to transmit boundary information in CAD/CFD conversion. By using the CAD Configuration Manager provided by ANSYS Workbench, the simulation platform can visit and modify the geometry file constructed by SolidWorks. The attributes attached by tags are used to guide the mesh generation as shown in Figure 13. Consequently, CAE boundary features are established, resulting in the generation of the fluid flow space, which is the input of the intelligent CFD solver.

According to the geometry and parameters automatically generated by the CAD system shown in Tables 2 and 3, they are stored in files or models in the form of text or expressions. The simulation part is implemented based on ANSYS Workbench. The platform provides two script levels. For application-level task automation, especially in CFX, the CFX Command Language (CCL) ("Workbench Scripting Guide" 2012) is used as a session to operate CFX-Pre and CFD-Post. Therefore, CCL can be used to perform physical model selection. After the simulation is completed, CCL can also automatically perform postprocessing. For project-level task automation, Workbench scripts ("Workbench Scripting Guide" 2013) is used to create the entire project and call various applications to complete the created project. Actions performed through the GUI are recorded as a log of Pythonbased scripts. Such scripts can be customized for specific purposes. Therefore, the function of the entire system has been greatly expanded without excessive scripting. Based on the provided tools, fluid physical characteristics and dynamic physical characteristics can be edited through the program CCL. In addition, the intelligent solver function is 
Inputs: midcurve $C_{k}$

Outputs: outflow gas domain $M_{c}$ and $M_{g}$

(1) $\mathrm{i} \leftarrow 0$, initialize and filter to group $L_{k}$ and $L_{k}^{\prime}$

(2) Loft them in order of height to get the midarc surface and extend the midarc surface $S_{m}$, blade number $N \leftarrow$ n, $\alpha_{\text {rotate }} \leftarrow 360^{\circ} / 2 \bullet N$, symmetrical rotating midarc $S_{m}$ to get $S_{m}^{\prime}$ and $S_{m}^{\prime \prime}$,

(3) Calculate the leaf pot, the dorsal periodic boundary, and the minimum bounding box of the leaf $B_{p}, B_{s}$, and $B_{\text {blade }}$, and then create the blade inclusion $E_{\text {cubic }}$,

(4) while $B_{\text {blade }} \subset B_{c}$ do

(5) if $B_{c} \in B_{p} \& \& B_{c} \in B_{s}$

(6) Using rotating midsurface $S_{m}^{\prime}$ and $S_{m}^{\prime \prime}$ to cut inclusions $E_{\text {cubic }}$,

(7) else adjust the size midsurface $S_{m}^{\prime}$ and $S_{m}^{\prime \prime}$

(8) Input the inlet and outlet gas angles $\alpha_{1}$ and $\alpha_{2}$, lengths $l_{1}$ and $l_{2}$, and stretch the entrance and exit surfaces of the package to generate the blade flow channel entity $M_{p}$,

(9) Subtract the blade solids $M_{b}$ and the cooling domain $M_{c}$ to get outflow gas domain $M_{g}$

(10) end while

(11) return $\left\{M_{c}, M_{g}\right\}$

Algorithm 2: Adaptive pipeline intersection algorithm.

installed into the CAD/CFD integrated system through Workbench scripts.

\section{Model Validation and Optimization Cases}

4.1. Numerical Method and Experimental Data Verification. Conjugated heat transfer (CHT) numerical simulation method was adopted to optimize the blade cooling structure. Further experimental data are required to verify method validity and find an appropriate numerical model.

The C3Xvane analytical and experimental evaluation has extensive experimental data under high pressure and temperature conditions.

The C3X experiment has detailed data. In this paper, the CHT method is used to simulate the C3X blade, and the simulation results are also compared. The data of blade geometry and experimental conditions 4521 are from the literature [41]. The calculation model contains a total of 12 computational domains: 1 gas domain, 1 solid domain, and 10 cooling air domains. The boundary layer grid is added to the fluid-solid interface to simulate the close avoidance. The $\mathrm{y}$-plus is less than 1 , and the inlet Mach number is equal to 0.17 , the total inlet temperature is $818 \mathrm{~K}$, and the gas-thermal coupling numerical simulation is solved in ANSYS CFX using the RANS method, and the SST turbulence model is applied.

The numerical simulation results were compared with the experimental data. According to Figure 14, the gasthermal coupling numerical simulation can well predict the pressure distribution on the blade surface. The SST turbulence model can give an accurate prediction of the temperature distribution near the leading edge, as shown in Figure 15. However, the CHT model tends to overestimate the convective heat transfer intensity after the suction side shock wave, so the calculated blade temperature is significantly higher than the experimental data. Besides, the calculation accuracy of the pressure surface is higher than the calculation accuracy of the suction surface because the suction phenomenon such as surface transition and flow separation occurs on the suction surface, and the full turbulence model is unable to predict the transition. Thus, using the SST turbulence model can provide an acceptable prediction of the cooling performance of the blade with an average error of less than $22.8 \mathrm{~K}$, and the SST model can give an acceptable prediction of convective heat transfer on the blade surface.

4.2. Multiphysics Model for CHTand FEA. This study focuses on improving the internal cooling efficiency, not paying much attention to the Tenon and platform design. Therefore, the Tenon and platform of the blade are simplified as a block, which could reduce the computational cost. At the same time, simulation parameters are preserved during the modeling process as equation (15) represented and the parameters through the custom script file transfer to ANSYS Workbench. CAD configuration manager provided by ANSYS Workbench can be used to update the blade geometry model parameters. Table 4 gives the optimization parameters' initial value, and Table 5 gives the film holes' design parameters and their design values.

The boundary conditions of the conjugate heat transfer are shown in Figure 16. The inlet total pressure is equal to 1.2 $\mathrm{MPa}$, the inlet total temperature is $1200 \mathrm{~K}$, and the flow direction is in the axial of the turbine engine. Coolant flow inlet mass flow rate is equal to $0.02 \mathrm{~kg} / \mathrm{s}$, and its total temperature is $650 \mathrm{~K}$. At the outlet, the static pressure is equal to $0.9 \mathrm{MPa}$.

\subsection{Optimization Design of Turbine Blade Film Hole and Cooling Mass Flow}

4.3.1. Direct Optimization. In this case, the optimal parameters of the film hole are set for the two optimization objectives $\eta_{1}$ and $\eta_{2}$ as introduced in Section 4.2, Table 4 .

Optimization variables can be expressed as follows:

$$
X *=\left[\alpha_{1}, \alpha_{2}, r, M\right],
$$




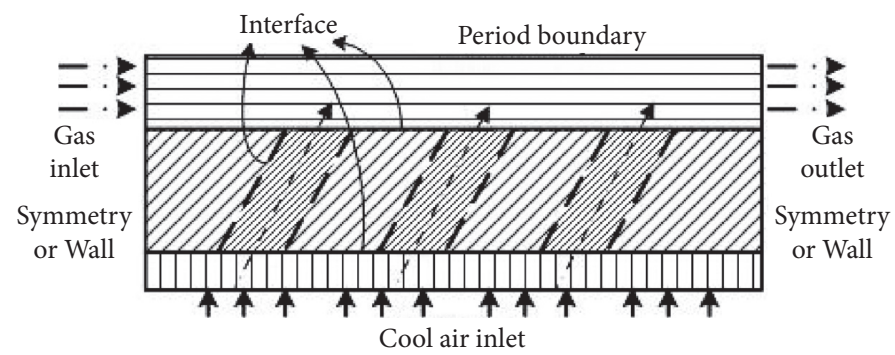

(a)

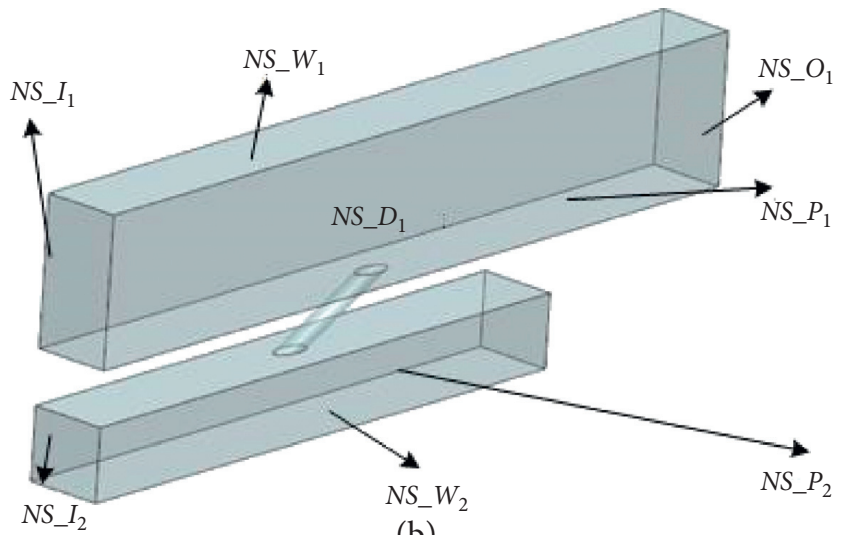

(b)

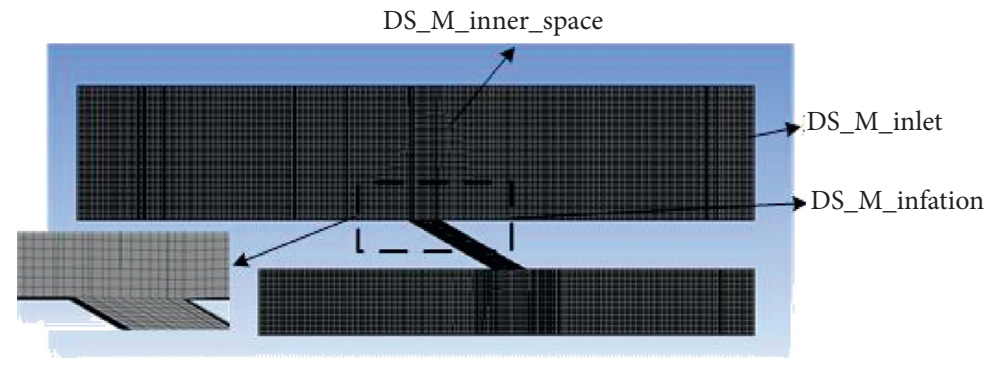

(c)

FIgURE 13: Model conversion in CAD and CFD.

TABLE 3: Multiphysics domain model parameters generated in CAD software for simulation.

\begin{tabular}{|c|c|c|}
\hline Tag and type & Attribute & Application \\
\hline 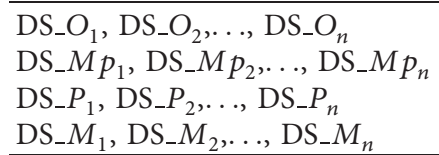 & $\begin{array}{l}\text { Optimization parameters } \\
\text { Material properties } \\
\text { Physical parameter } \\
\text { Mesh type } \\
\end{array}$ & $\begin{array}{c}\text { DOE and optimization } \\
\text { Simulation or optimization } \\
\text { Simulation boundary condition } \\
\text { Simulation }\end{array}$ \\
\hline
\end{tabular}

where $\alpha_{1}$ is film hole row 1and row 2 composition angle on blade suction surface, $\alpha_{2}$ is film hole row 3 and row 4 composition angle on blade pressure surface, $r$ is film hole radius, and $q$ is cooling air mass flow.

Figure 17 shows the schematic design of film hole-related parameters and conversion relationship between the film hole design angles. In order to save computing space and improve optimization efficiency, response surface methods (RSMs) with Latin hypercube sampling design is dedicated to approximate the empirical relationship between the objective function and the design variable. Table 6 shows twenty-five design points and design variables.

For the two objective functions in equations (23) and (24) and one constraint function equation (26), the optimization problem is expressed as the following mathematical model:

$$
\min F(X *)=\left\{\eta_{1},-\eta_{2}\right\} \text { S.t. } g_{m}(X *) \leq 0 \sigma_{\max } \leq 0.8 * \sigma_{0.2}^{T} \text {. }
$$

Optimization results are shown in Table 7.

Compared with the original design point, maximum temperature and average temperature of the blade are reduced with little promotion of cooling air mass flow. The overall cooling efficiency is improved with the constraint that the yield strength is under $0.8 * \sigma_{0.2}^{T}(750 \mathrm{MPa})$. Each of the above examples takes only about 4 hours from design, 


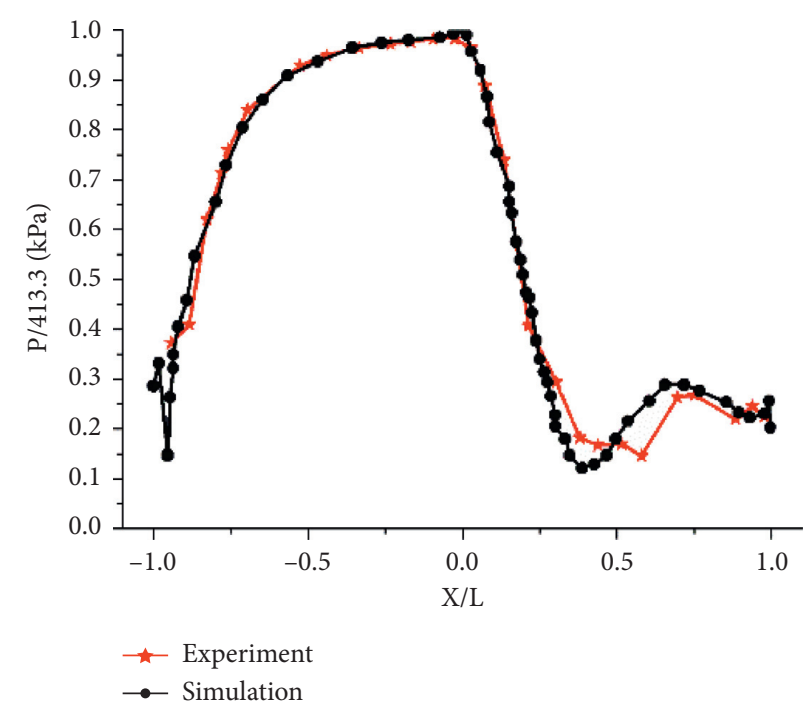

Figure 14: Pressure distribution on the midspan section of the blade surface.

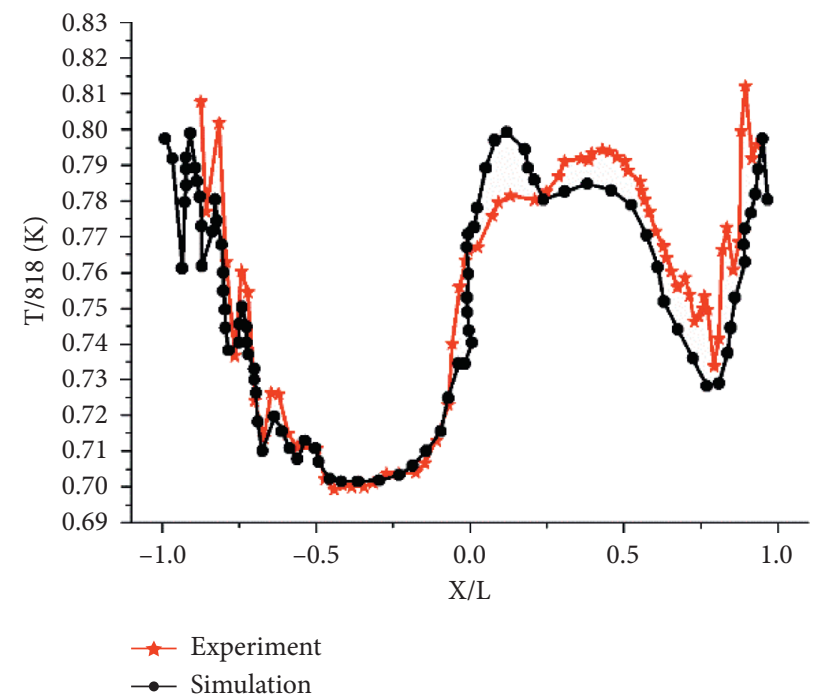

FIGURE 15: Temperature distribution on the midspan section of the blade surface.

TABLE 4: Optimization parameters' initial value.

\begin{tabular}{lcccc}
\hline Optimization parameters & $\eta_{1}$ & $T_{g, r}(K)$ & $T_{a w}(K)$ & $T_{c}(K)$ \\
\hline Initial value & $72.72 \%$ & 1200 & 800 & 750 \\
\hline
\end{tabular}

modeling to simulation, which meets engineering design requirements.

4.3.2. Indirect Optimization. Through the generation of summary of cooling structure introduced in Section 4.2, a simplified cooling model can also be generated for batch optimization. Due to the relationship between curved and twisted blades, when the angle range of the gas film hole is too large, not only is it difficult to model, but also difficult to
TABLE 5: Film holes' design parameters and their design values.

\begin{tabular}{lcccccc}
\hline Design parameters & $\eta_{f}$ & $k$ & $c$ & $X(\mathrm{~mm})$ & $M$ & $S(\mathrm{~mm})$ \\
\hline Design value & $27.2 \%$ & $k_{f}$ & $c_{f}$ & 1 & 2 & 0.4 \\
\hline
\end{tabular}

process, and the gas film cooling effect reaches a certain degree corresponding to a specific injection angle range, so the cooling effect can be performed first. The gas film hole can be simplified first, and the cooling channel is simplified to a straight channel, thereby reducing the range of design parameters.

The main parameters of the blade film hole are extracted according to the method in Section 3.3. The gas film hole cooling performance was studied, and the gas film cooling angle and the length of the gas film hole were determined. 


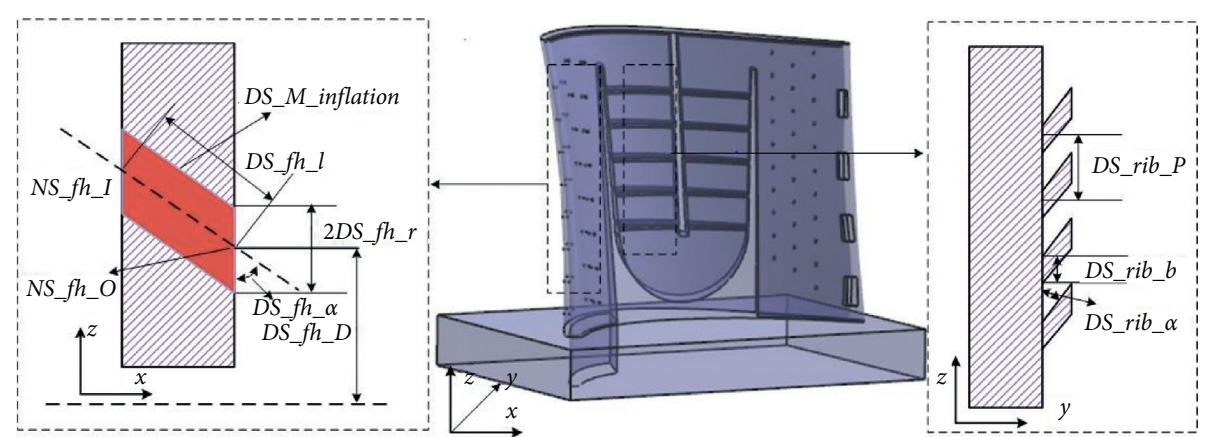

(a)

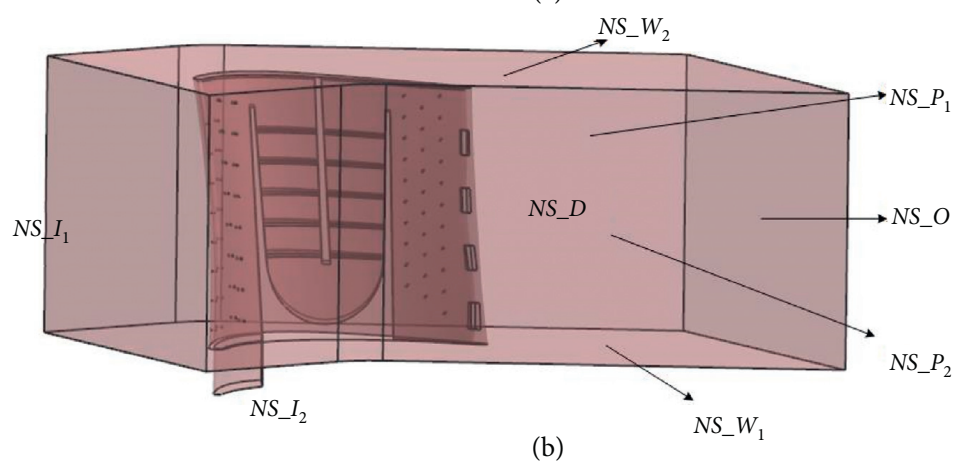

(b)

FIGURE 16: Multiphysics model and simulation parameters.

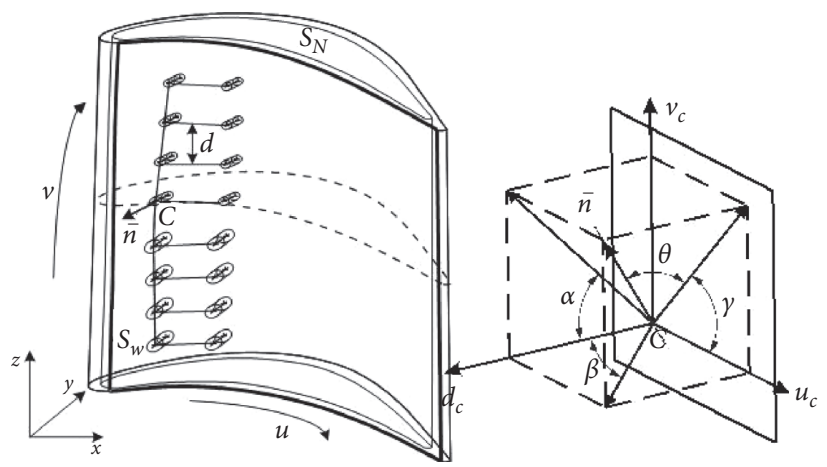

FIgURE 17: Schematic design of film hole-related parameters and conversion relationship between the film hole design angles.

Due to the fixed blade thickness, when the gas film hole angle was determined, the length of the gas film hole was also determined. Boundary conditions intercept the main boundary conditions of the blade.

Latin hypercube is a sampling method that is widely used in optimization, especially in approximate simulation calculations where the position of the sample point data in the entire design space is high. It can be implemented by the LHS design command in MATLAB. Parameterization mainly uses the script file of ANSYS ICEM to realize the call to ICEM software. The final parameterization program can generate a .cfx 5 format file, which can be directly read by the CFX preprocessing macro file. In order to reduce the amount of calculation in the optimization process, this paper also introduces the RBNN neural network commonly used in approximate models in the optimization system, which can be implemented by MATLAB commands. The optimization algorithm selects the genetic algorithm that researchers mostly use. Through the control of the main program, the above modules are combined, and the optimal cooling structure can be obtained directly for the given aerodynamic conditions, optimized design variables, and optimized goals. The entire process does not require human intervention. The jet angle of the gas film hole is an important factor affecting its cooling performance. Reducing the jet angle will reduce the velocity component of the cooling air flow perpendicular to the cooling surface, and the effect of the air flow infiltrating into the mainstream will be weakened. More cooling air flow is distributed on the cooling surface, and the cooling air flow can extend 
TABLE 6: Design points and its variables.

\begin{tabular}{|c|c|c|c|c|}
\hline Design points & $\alpha_{1}$ & $\alpha_{2}$ & $d$ & $q$ \\
\hline DP1 & -87.3 & 120.7 & 0.11 & 0.046 \\
\hline DP2 & -60.3 & 118.9 & 0.1388 & 0.06 \\
\hline DP3 & -53.1 & 108.1 & 0.0236 & 0.05 \\
\hline DP4 & -65.7 & 117.1 & 0.1244 & 0.072 \\
\hline DP5 & -78.3 & 135.1 & 0.038 & 0.034 \\
\hline DP6 & -80.1 & 138.7 & 0.1892 & 0.056 \\
\hline DP7 & -58.5 & 136.9 & 0.1172 & 0.062 \\
\hline DP8 & -63.9 & 122.5 & 0.182 & 0.038 \\
\hline DP9 & -76.5 & 131.5 & 0.074 & 0.064 \\
\hline DP10 & -45.9 & 144.1 & 0.0596 & 0.036 \\
\hline DP11 & -89.1 & 102.7 & 0.0884 & 0.03 \\
\hline DP12 & -69.3 & 124.3 & 0.0524 & 0.07 \\
\hline DP13 & -74.7 & 113.5 & 0.1028 & 0.068 \\
\hline DP14 & -51.3 & 104.5 & 0.146 & 0.044 \\
\hline DP15 & -81.9 & 140.5 & 0.0812 & 0.074 \\
\hline DP16 & -56.7 & 115.3 & 0.1676 & 0.042 \\
\hline DP17 & -71.1 & 142.3 & 0.1604 & 0.04 \\
\hline DP18 & -49.5 & 106.3 & 0.1316 & 0.052 \\
\hline DP19 & -72.9 & 109.9 & 0.0452 & 0.028 \\
\hline DP20 & -47.7 & 111.7 & 0.0308 & 0.058 \\
\hline DP21 & -62.1 & 133.3 & 0.0956 & 0.054 \\
\hline DP22 & -85.5 & 100.9 & 0.1748 & 0.066 \\
\hline DP23 & -54.9 & 126.1 & 0.1964 & 0.048 \\
\hline DP24 & -83.7 & 129.7 & 0.0668 & 0.032 \\
\hline DP25 & -67.5 & 127.9 & 0.1532 & 0.026 \\
\hline
\end{tabular}

TABLE 7: Optimization results.

\begin{tabular}{lcccccc}
\hline Design point & $\eta_{1}(\%)$ & $\eta_{2}(\%)$ & $\sigma_{\max }(\mathrm{MPa})$ & $T_{\max }(\mathrm{K})$ & $T_{\text {ave }}(\mathrm{K})$ & $q(\mathrm{~kg} \mathrm{~s})$ \\
\hline ODP & 79.47 & 98 & 762 & 910 & 762 & 0.04 \\
CDP1 & 82.53 & 54 & 748 & 861 & 746 & 0.0525 \\
CDP2 & 82.49 & 36 & 744 & 857 & 746 & 0.0536 \\
CDP3 & 82.30 & 38 & 740 & 851 & 747 & 0.056 \\
\hline
\end{tabular}

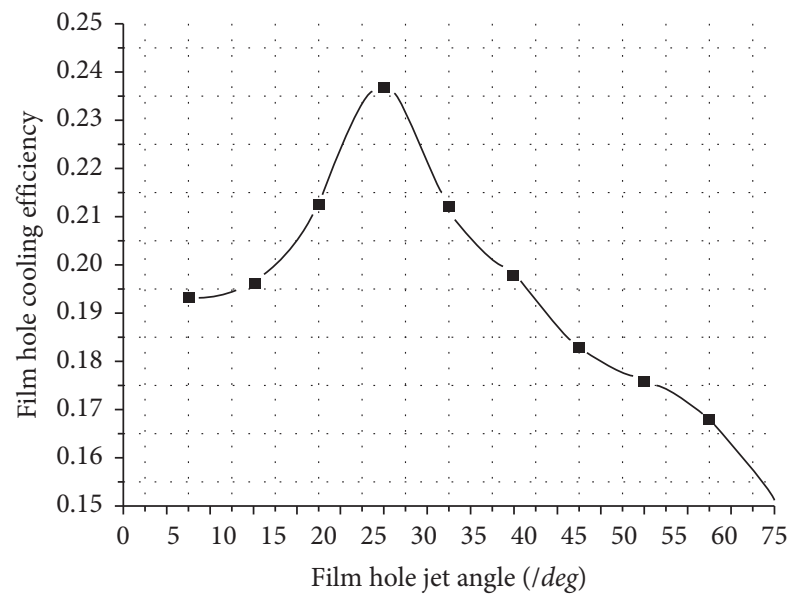

(a)

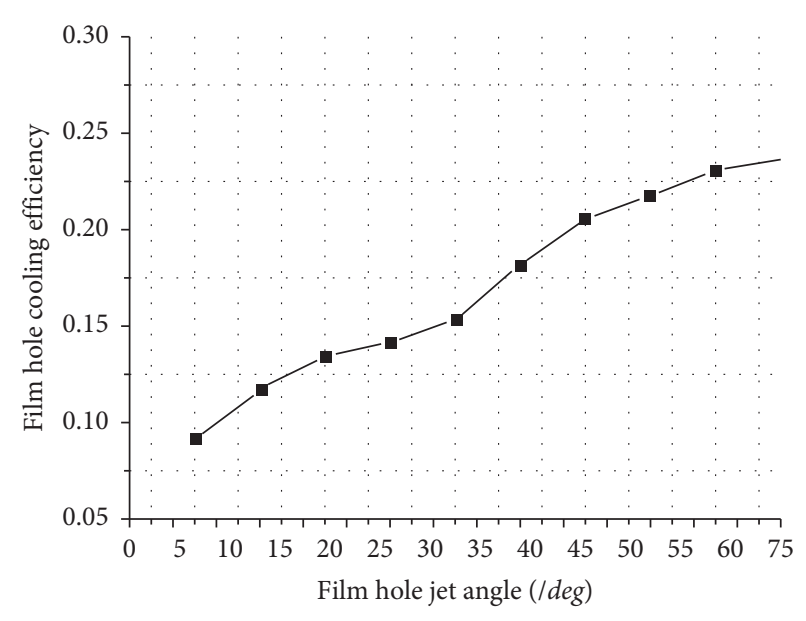

(b)

Figure 18: The effect of the compound angle on the average film hole cooling efficiency of the film hole area. 
TABle 8: Optimization results.

\begin{tabular}{lcc}
\hline Parameters & Optimization results (design range) & Units \\
\hline DS_rib_e & $1.75(0.5-2)$ & $\mathrm{Mm}$ \\
DS_rib_P & $5.94(5-10)$ & $\mathrm{Mm}$ \\
DS_rib_ $\alpha$ & $59.6(50-130)$ & Deg \\
\hline
\end{tabular}

further away in the flow direction. However, in actual blades, due to the constraints of structure and manufacturing, it is impossible to produce gas film holes with a very small jet angle.

As shown in Figure 18, it can be seen that the film cooling efficiency increases with the increase of the compound angle. The increase in amplitude indicates that the compound angle has a greater effect at high blowing ratios. After the recombination angle is greater than $45^{\circ}$, the cooling efficiency of the gas film hole at a high blowing ratio becomes higher than that at a low blowing ratio, indicating that a large composite angle can fully exert the cooling capacity of the cooling air flow at a high blowing ratio.

According to the analysis results, it can be concluded that the gas film cooling efficiency should reach 0.2 , and the injection angle and composite angle range are $(18,45)$ and $(45,75)$.

Simplify the cooling channel of the blade, through the parametric modeling module, enter the cooling channel parameters, and the simplified geometric model and analysis model are shown in the figure. The design range and optimization results of design parameters are shown in Table 8 .

By optimizing the range of film cooling parameters and the optimization of cooling channels, the blades are optimized. The film cooling range has been effectively improved. The average cooling effect of the blade body cooling channel is better than the initial design.

\section{Conclusions}

(1) According to the model adaptability problem of complex product model in multiphysics simulation, the differences of model expression methods in design, modeling, and simulation were analyzed to improve the adaptability of complex product models to multiphysics simulation.

(2) The product design space and analytical space mapping method adapted to multiphysics simulation was studied. According to the existing principles and empirical formulas, the relationship between design parameters modeling parameters and simulation parameters was established by unified CADSS parameters, so that the analysis results can be quickly fed back to the modification of geometric models to break the isolation between design and analysis.

(3) According to the analysis and optimization requirements, blade model for CHT and FEA simulation was built from the top down under the CADSS framework. The multiphysics blade model containing simulation parameters adapts well to multidisciplinary simulation and can generate batch design point models for optimization.

(4) Through the blade's MDM by CADSS and optimization design of the film hole, 25 design points were generated for about 2 hours for each case which takes more time through conventional design and simulation process. Three candidate design points were chosen by MOGA and effectively promoted cooling efficiency with less promotion of cooling mass flow. The case studied in the paper shows the method proposed can improve design efficiency and shorten the design cycle.

\section{Data Availability}

The data that support the findings of the research are available from the corresponding author.

\section{Conflicts of Interest}

The authors declare that there are no conflicts of interest regarding the publication of this paper.

\section{References}

[1] M. S. Shephard, M. W. Beall, R. M. O'bara, and B. E. Webster, "Toward simulation-based design," Finite Elements in Analysis and Design, vol. 40, no. 12, pp. 1575-1598, 2004.

[2] Y. Zeng and I. Horváth, "Fundamentals of next generation CAD/E systems," Computer-Aided Design, vol. 44, no. 10, pp. 875-878, 2012.

[3] Y. Bodein, B. Rose, and E. Caillaud, "Explicit reference modeling methodology in parametric CAD system," Computers in Industry, vol. 65, no. 1, pp. 136-147, 2014.

[4] M. S. Smit and W. F. Bronsvoort, "Integration of design and analysis models," Computer-Aided Design and Applications, vol. 6, no. 6, pp. 795-808, 2009.

[5] S. Ando, H. Aoyama, and N. Sano, CAD System for Utilizing Design Information and Know-How Based on Function Features, American Society of Mechanical Engineers (ASME), London, UK, 2012.

[6] F. Tian and M. Voskuijl, "Automated generation of multiphysics simulation models to support multidisciplinary design optimization," Advanced Engineering Informatics, vol. 29, no. 4, pp. 1110-1125, 2015.

[7] J. R. R. A. Martins and A. B. Lambe, "Multidisciplinary design optimization: a survey of architectures," AIA A Journal, vol. 51, no. 9, pp. 2049-2075, 2013.

[8] X.-J. Ma, G.-F. Ding, S.-F. Qin et al., "Transforming multidisciplinary customer requirements to product design specifications," Chinese Journal of Mechanical Engineering, vol. 30, no. 5, pp. 1069-1080, 2017.

[9] O. Hamri, J. C. Léon, F. Giannini et al., "Software environment for CAD/CAE integration," Advances in Engineering Software, vol. 41, no. 10-11, pp. 1211-1222, 2010.

[10] Z. Chi, J. Ren, and H. Jiang, "Coupled aerothermodynamics optimization for the cooling system of a turbine vane," Journal of Turbomachinery, vol. 136, no. 5, 2014.

[11] Q. Li, P. Liu, and G. He, "Fluid-solid coupled simulation of the ignition transient of solid rocket motor," Acta Astronautica, vol. 110, pp. 180-190, 2015. 
[12] B. Chen and Y. Xie, "A computational approach for the optimal conceptual design synthesis based on the distributed resource environment," International Journal of Production Research, vol. 55, no. 20, pp. 5881-5901, 2017.

[13] X. Jiao, G. Zheng, P. A. Alexander et al., "A system integration framework for coupled multiphysics simulations," Engineering with Computers, vol. 22, no. 3-4, pp. 293-309, 2006.

[14] B. C. Kim and D. Mun, "Feature-based simplification of boundary representation models using sequential iterative volume decomposition," Computers \& Graphics, vol. 38, pp. 97-107, 2014.

[15] G. P. Gujarathi and Y.-S. Ma, "Parametric CAD/CAE integration using a common data model," Journal of Manufacturing Systems, vol. 30, no. 3, pp. 118-132, 2011.

[16] N. Aifaoui, D. Deneux, and R. Soenen, "Feature-based interoperability between design and analysis processes," Journal of Intelligent Manufacturing, vol. 17, no. 1, pp. 13-27, 2006.

[17] M. S. Shephard, P. L. Baehmann, M. K. Georges et al., "Framework for the reliable generation and control of analysis idealizations," Computer Methods in Applied Mechanics and Engineering, vol. 82, no. 1-3, pp. 257-280, 1990.

[18] S. H. Lee, "A CAD-CAE integration approach using featurebased multi-resolution and multi-abstraction modelling techniques," Computer-Aided Design, vol. 37, no. 9, pp. 941-955, 2005.

[19] B. A. Szabó, "Geometric idealizations in finite element computations," Communications in Applied Numerical Methods, vol. 4, no. 3, pp. 393-400, 1988.

[20] N. Aifaoui, D. Deneux, A. Benamara et al., "Mechanical analysis process modeling based on analysis features," IEEE, vol. 3, p. 6, 2002.

[21] Z. Wang, L. Tian, and W. Duan, "Annotation and retrieval system of CAD models based on functional semantics," Chinese Journal of Mechanical Engineering, vol. 27, no. 6, pp. 1112-1124, 2014.

[22] Z. Pan, X. Wang, R. Teng, and X. Cao, "Computer-aided design-while-engineering technology in top-down modeling of mechanical product," Computers in Industry, vol. 75, pp. 151-161, 2016.

[23] V. Shapiro, I. Tsukanov, and A. Grishin, "Geometric issues in computer aided design/computer aided engineering integration," Journal of Computing and Information Science in Engineering, vol. 11, no. 2, 2011.

[24] I. Matin, M. Hadzistevic, J. Hodolic et al., "A CAD/CAEintegrated injection mold design system for plastic products," The International Journal of Advanced Manufacturing Technology, vol. 63, no. 5-8, pp. 595-607, 2012.

[25] R. S. Peak, R. E. Fulton, I. Nishigaki, and N. Okamoto, "Integrating engineering design and analysis using a multirepresentation approach," Engineering with Computers, vol. 14, no. 2, pp. 93-114, 1998.

[26] R. Sudarsan, S. J. Fenves, R. D. Sriram, and F. Wang, “A product information modeling framework for product lifecycle management," Computer-Aided Design, vol. 37, no. 13, pp. 1399-1411, 2005.

[27] L. Li, C. F. Lange, and Y. Ma, "Association of design and computational fluid dynamics simulation intent in flow control product optimization," Proceedings of the Institution of Mechanical Engineers, Part B: Journal of Engineering Manufacture, vol. 232, no. 13, pp. 2309-2322, 2018.

[28] M.-C. Hsu, C. Wang, A. J. Herrema, D. Schillinger, A. Ghoshal, and Y. Bazilevs, "An interactive geometry modeling and parametric design platform for isogeometric analysis," Computers \& Mathematics with Applications, vol. 70, no. 7, pp. 1481-1500, 2015.

[29] A. J. Herrema, N. M. Wiese, C. N. Darling, B. Ganapathysubramanian, A. Krishnamurthy, and M.-C. Hsu, "A framework for parametric design optimization using isogeometric analysis," Computer Methods in Applied Mechanics and Engineering, vol. 316, pp. 944-965, 2017.

[30] E. Ferede, M. M. Abdalla, and G. J. W. van Bussel, "Isogeometric based framework for aeroelastic wind turbine blade analysis," Wind Energy, vol. 20, no. 2, pp. 193-210, 2017.

[31] P. Kang and S.-K. Youn, "Isogeometric topology optimization of shell structures using trimmed NURBS surfaces," Finite Elements in Analysis and Design, vol. 120, pp. 18-40, 2016.

[32] X. Li, C. D. LeiWei, and W. WangLi, "Statistical process monitoring with biogeography-based optimization independent component analysis," Mathematical Problems in Engineering, vol. 2018, no. 1, pp. 1-14, 2018.

[33] J. Li, T. Ning, T. Wang, B. Hu, P. Xi, and J. Xu, "A rapid parameter configuration method for film hole component in pipe-net calculation," Proceedings of the Institution of Mechanical Engineers, Part A: Journal of Power and Energy, vol. 234, no. 7, pp. 915-933, 2020.

[34] C. Li, A Two-Dimensional Analytical Method for Turbine Blade Cooling Design, American Society of Mechanical Engineers (ASME), London, UK, 2017.

[35] Z. Cheng and Y. Ma, "Explicit function-based design modelling methodology with features," Journal of Engineering Design, vol. 28, no. 3, pp. 205-231, 2017.

[36] J. Li, T. Ning, P. Xi, B. Hu, and T. Wang, "An analysis-oriented parameter extraction method for features on freeform surface," Proceedings of the Institution of Mechanical Engineers, Part C: Journal of Mechanical Engineering Science, vol. 233, no. 17, pp. 6005-6025, 2019.

[37] F. Wagner, "Multi-objective optimization of the cooling configuration of a high pressure turbine blade," American Society of Mechanical Engineers (ASME), London, UK, 2018.

[38] R. J. Goldstein, "Film cooling," Advances in Heat Transfer, vol. 23, pp. 321-379, 1971.

[39] X. Wang, H. Xu, J. Wang, W. Song, and M. Wang, "Multiobjective optimization of discrete film hole arrangement on a high pressure turbine end-wall with conjugate heat transfer simulations," International Journal of Heat and Fluid Flow, vol. 78, 2019.

[40] Z. Wang, W.-J. C. Du, and S.-J. Li, "Multi-field coupling analysis on the film-cooling with transverse and arched trenches," Journal of Thermal Science and Technology, vol. 14, no. 1, 2019.

[41] L. D. Hylton, M. S. Mihelc, E. R. Turner et al., "Analytical and Experimental Evaluation of the Heat Transfer Distribution over the Surfaces of Turbine vanes," 1983. 